\title{
Autonomous Demand Response for Primary Frequency Regulation
}
M Donnelly
S Mattix
D Trudnowski
JE Dagle

January 2012

Pacific Northwest

NATIONAL LABORATORY

Proudly Operated by Battelle Since 1965 


\title{
DISCLAIMER
}

This report was prepared as an account of work sponsored by an agency of the United States Government. Neither the United States Government nor any agency thereof, nor Battelle Memorial Institute, nor any of their employees, makes any warranty, express or implied, or assumes any legal liability or responsibility for the accuracy, completeness, or usefulness of any information, apparatus, product, or process disclosed, or represents that its use would not infringe privately owned rights. Reference herein to any specific commercial product, process, or service by trade name, trademark, manufacturer, or otherwise does not necessarily constitute or imply its endorsement, recommendation, or favoring by the United States Government or any agency thereof, or Battelle Memorial Institute. The views and opinions of authors expressed herein do not necessarily state or reflect those of the United States Government or any agency thereof.

\author{
PACIFIC NORTHWEST NATIONAL LABORATORY \\ operated by \\ BATTELLE \\ for the \\ UNITED STATES DEPARTMENT OF ENERGY \\ under Contract DE-AC05-76RL01830 \\ Printed in the United States of America \\ Available to DOE and DOE contractors from the \\ Office of Scientific and Technical Information, \\ P.O. Box 62, Oak Ridge, TN 37831-0062; \\ ph: (865) 576-8401 \\ fax: (865) 576-5728 \\ email: reports@adonis.osti.gov
}

\author{
Available to the public from the National Technical Information Service, \\ U.S. Department of Commerce, 5285 Port Royal Rd., Springfield, VA 22161 \\ ph: (800) 553-6847 \\ fax: (703) 605-6900 \\ email: orders@ntis.fedworld.gov \\ online ordering: http://www.ntis.gov/ordering.htm
}

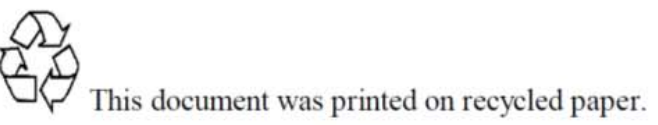

(9/2003) 


\title{
Autonomous Demand Response for Primary Frequency Regulation
}

\author{
M Donnelly $^{1} \quad$ S Mattix ${ }^{1}$ \\ D Trudnowski $^{1} \quad$ JE Dagle
}

January 2012

Prepared for

U.S. Department of Energy

under Contract DE-AC05-76RL01830

Pacific Northwest National Laboratory

Richland, Washington 99352

\footnotetext{
${ }^{1}$ Montana Tech
} 



\section{Executive Summary}

Demand response has long been an integral part of power system control and operation. Recently, demand response has received more interest as a potentially effective tool to help gain higher levels of asset utilization on the bulk power grid and to avoid or delay the need for new transmission-line construction.

Autonomous demand response is defined as load response to system-based signals rather than to master control signals or price signals from a central dispatch center. The most readily available systembased signal is frequency, which can be a very reliable indicator of grid instability, problems, or abnormal conditions. Speed governing systems at central generating stations, when operating in droop mode, employ frequency as the primary feedback signal. These governors are largely responsible for affecting primary frequency response of the bulk power grid thereby maintaining a continuous balance between supply and demand.

This research examines the use of autonomous demand response to provide primary frequency response in an interconnected grid. Ultimately, it is conceivable that all primary frequency response might be delivered by responsive load leaving dispatchable generation to be base loaded or ramped with very slow ramp rates. If this objective were to be achieved, numerous benefits might be realized, including reduced emissions from fossil plants as a result of higher operating efficiencies and greater flexibility in integrating variable generation sources such as wind and solar. The work builds on previous studies in several key areas: it uses a large realistic model (i.e., the interconnection of the western United States and Canada); it establishes a set of metrics that can be used to assess the effectiveness of autonomous demand response; and it independently adjusts various parameters associated with using autonomous demand response to assess effectiveness and to examine possible threats or vulnerabilities associated with the technology. Where prior research has focused on showing the efficacy of the concept in delivering primary frequency response, this study's primary objective was to identify any potential deleterious effects.

More than 6000 simulations of the power system model were conducted during the course of the study. A key finding is that there are very few conditions associated with autonomous demand response that have the potential to degrade reliability. Substantial improvement in primary frequency response was demonstrated in almost all cases without negative impacts on other aspects of system reliability. Two areas of concern documented in the study are excessive time delay within the control loop and high penetration of autonomous demand response concentrated in one region of an interconnected grid. Both concerns are related to oscillatory stability of the grid, not with frequency response, and both could be corrected by appropriate design of the control law. The authors conclude that frequency-based autonomous demand response should not be used in conjunction with demand-side appliances that respond slowly to frequency excursions unless care is taken to ensure that the control law takes into account the appropriate delay.

Another finding is that the marginal benefit attributable to autonomous demand response is quantifiable, and can be used to determine the value of the technology for providing primary frequency response in an environment of increasing costs for this service as provided by traditional means. Additional work is needed to verify the findings of this preliminary investigation. It is proposed that this work be conducted in collaboration with the electric utility industry. 


\section{Acknowledgments}

The authors would like to acknowledge the generous support of Pacific Northwest National

Laboratory, the Western Electricity Coordinating Council, and the U.S. Department of Energy. 


\section{Terms, Acronyms, and Abbreviations}

area control error (ACE)

automatic generation control (AGC)

California-Oregon Interchange (COI)

California-Oregon Intertie (COI)

contingency

contingency reserve

d.c.

demand response
The instantaneous difference between a balancing authority's net actual and scheduled interchange, taking into account the effects of frequency bias [1].

Equipment that automatically adjusts generation in a balancing authority area from a central location to maintain the balancing authority's interchange schedule plus frequency bias. AGC also may accommodate automatic inadvertent payback and time error correction [1].

A flowgate (defined below) defined at the California-Oregon border. Not to be confused with California-Oregon Intertie.

A set of power lines at $500 \mathrm{kV}$ lines that constitute Path 66 in the WECC Path Rating Catalog.

The unexpected failure or outage of a system component, such as a generator, transmission line, circuit breaker, switch or other electrical element [1].

The provision of capacity deployed by the balancing authority to meet the disturbance control standard (defined below) and other North American Electric Reliability Corporation (defined below) and regional reliability organization contingency requirements [1].

direct current

The Federal Energy Regulatory Commission defines demand response as the ability of customers to respond to either a reliability trigger or a price trigger from their utility system operator, load-serving entity, regional transmission organization/independent system operator, or other demand response provider by lowering their power consumption [2]. In this report, the term is more generally defined as the ability of customers to respond to a reliability trigger or a price trigger from their utility system operator, load-serving entity, regional transmission organization/independent system operator, or other demand response provider, or to respond to a reliability trigger based on local measurements of system or exogenous conditions, by lowering their power consumption.

Federal Energy Regulatory Commission (www.ferc.gov)

FERC 
flowgate

frequency bias

frequency nadir

frequency regulation

frequency response

frequency response characteristic (FRC)

frequency responsive reserve (FRR)
A mathematical construct, comprised of one or more monitored transmission facilities and optionally one or more contingency facilities. Used to analyze the impact of power flows upon the bulk electric system [1].

A value, usually expressed in megawatts per $0.1 \mathrm{Hertz}$ $(\mathrm{MW} / 0.1 \mathrm{~Hz})$, associated with a balancing authority area that approximates the balancing authority area's response to Interconnection frequency error [1].

The minimum system frequency reached during the period of primary frequency control as the power system is responding to a step-change imbalance in energy supply and demand. It is sometimes difficult to determine the frequency nadir when observing only one system location; therefore, the frequency nadir is determined by averaging and low-pass filtering the location frequency at several generating stations throughout the system.

The ability of a balancing authority to help the Interconnection maintain scheduled frequency. This assistance can include both turbine governor response and automatic generation control [1].

Equipment - The ability of a system or elements of the system to react or respond to a change in system frequency. System - The sum of the change in demand, plus the change in generation, divided by the change in frequency, expressed in megawatts per $0.1 \mathrm{Hertz}(\mathrm{MW} / 0.1 \mathrm{~Hz})$ [1].

The natural characteristic of a balancing authority by which the generation output and load responds to deterioration of frequency. The unit of measure for FRC is percent per 0.1 $\mathrm{Hz}$, where the percent refers to the portion of the online generating capacity and load that has responded to the frequency disturbance. This may also be estimated by the megawatt change in the balancing authority interconnection flow [3].

That portion of operating reserve that responds to deterioration of frequency. The unit of measure for FRR is megawatts. It is common for resources to have some dead band (typically $0.036 \mathrm{~Hz}$ ) within which there is no response to deterioration of frequency [3]. 
governor droop

heavy spring (HSP)

heavy winter (HW)

Inhibit of Restore

LBNL

light summer (LS)

Localization

NERC

operating reserve

primary frequency control

PSLF

secondary frequency control
The characteristic by which a generator governor causes the output of the generator to change in response to a change in frequency. The Western Electricity Coordinating Council (defined below) currently requires the governor droop setting to be 5 percent [3].

Base case involving significant generation from hydroelectric resources in the Pacific Northwest.

Base case involving significant heating loads in cold regions of the planning domain.

Term refers to a characteristic of many end use appliances where, once turned off, it may not be practical to immediately restore power to the affected part of these appliances.

Lawrence Berkeley National Laboratory

Base case involving a situation in which maintaining an acceptable voltage profile might be a challenge.

Term used in this study to address a common and valid issue regarding autonomous demand response programs.

North American Electric Reliability Corporation (www.nerc.com)

That capability above firm system demand required to provide for regulation, load-forecasting error, equipmentforced and scheduled outages, and local-area protection. It consists of spinning and non-spinning reserves [1].

The turbine governor response portion of frequency regulation. Primary frequency control relates closely to frequency response.

\section{Positive Sequence Load Flow}

As opposed to primary frequency control, secondary frequency control involves slower, centrally (i.e., externally) directed actions that affect frequency more slowly than primary control (i.e., in tens of seconds to minutes). AGC is an automatic form of secondary frequency control [4]. 
settling frequency

spinning reserve

system droop

threat

tie line

WECC
The frequency to which the interconnected system converges following the period of primary frequency response. It is sometimes difficult to determine the settling frequency when observing only one system location. Therefore, the settling frequency is determined by averaging and low-pass filtering the frequencies at several generating stations throughout the system and then choosing a point at which the frequency has stabilized such that successive measurements of system frequency yield only a small deviation, or $\mathrm{dF} / \mathrm{dt}$ is less than the threshold value.

Unloaded generation that is synchronized and ready to serve additional demand [1].

The characteristic by which a system, balancing authority, or other aggregation of loads and resources respond to a change in frequency in the absence of an AGC action. For underfrequency events, the typical average system droop for the Western Electricity Coordinating Council is about 15 percent, which is equivalent to a system with one-third of the online units responsive to frequency with a 5 percent governor droop [3].

A system vulnerability that results (possibly inadvertently) from the action of the demand response system. The term is not intended to signify a deliberate cyber (or other) attack.

A circuit connecting two balancing authority areas [1].

Western Electricity Coordinating Council (www.wecc.biz). 


\section{Contents}

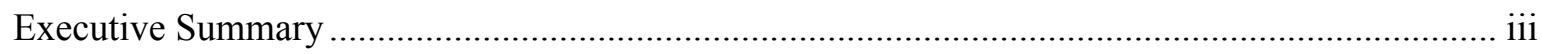

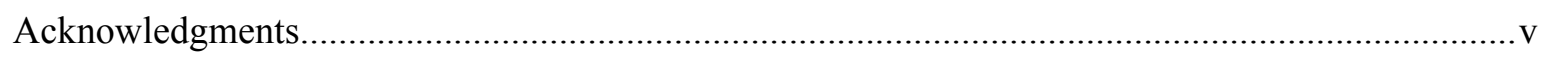

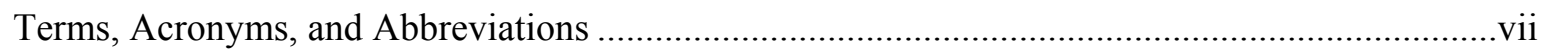

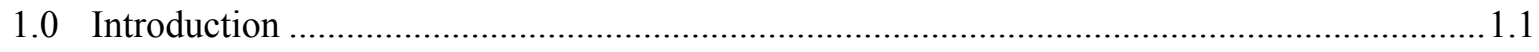

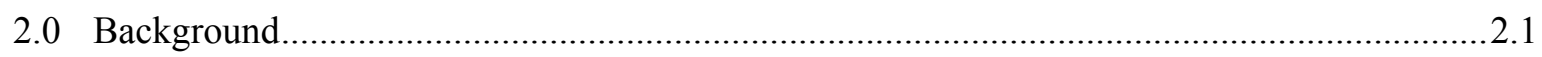

3.0 Power System Model and Modeling Assumptions................................................................. 3.1

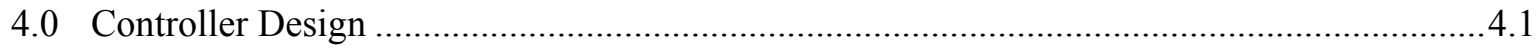

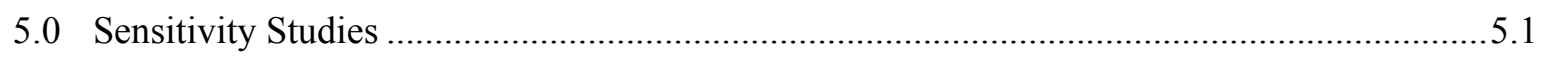

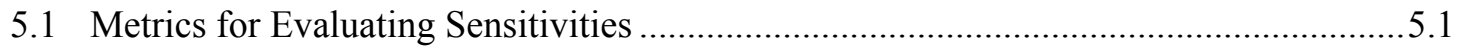

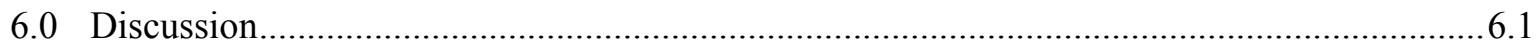

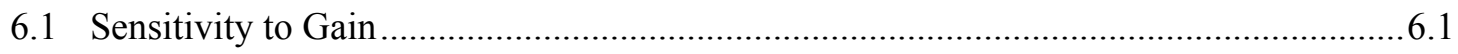

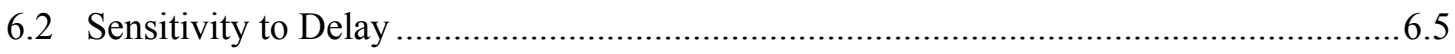

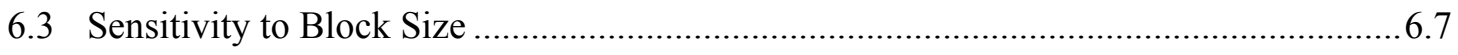

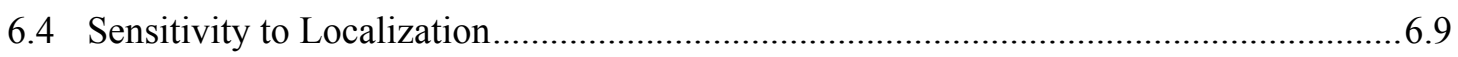

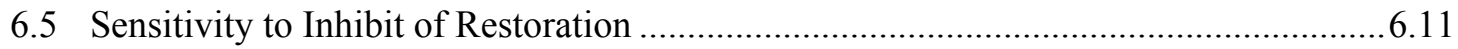

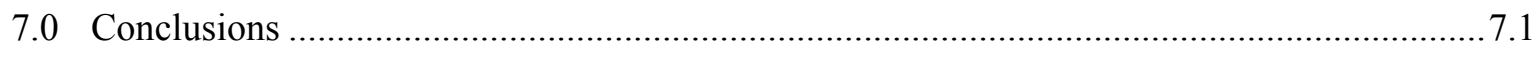

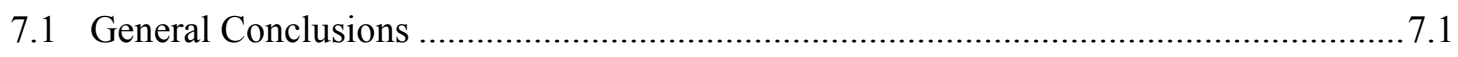

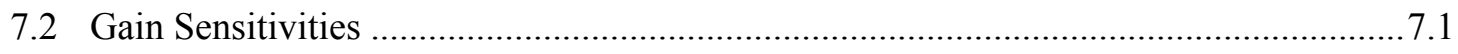

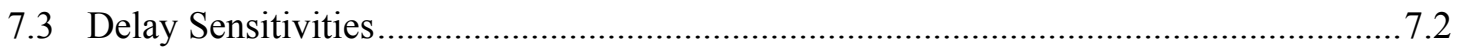

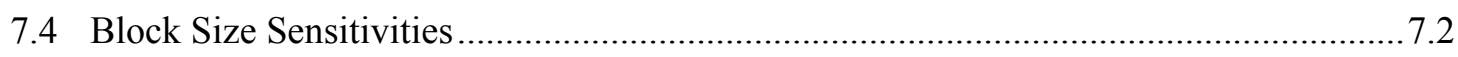

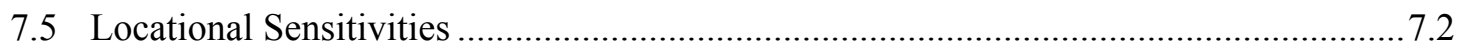

7.6 Inhibit Load Restoration Sensitivities .................................................................. 7.3

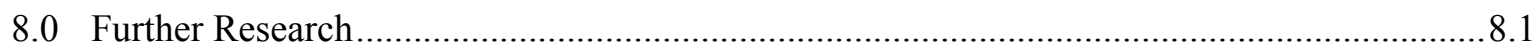

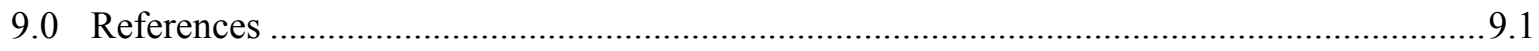

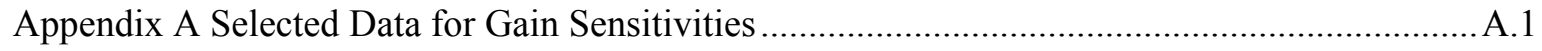

Appendix B Sample Source Code for In-Run EPCL ........................................................... B.1 


\section{Figures}

Figure 1. Transfer Capabilities between Various Regions of the WECC ................................

Figure 2. Controller Block Diagram ................................................................................. 4.1

Figure 3. Example of Frequency at Two Different System Locations................................. 4.2

Figure 4. Basis for Frequency Response Metrics ............................................................ 5.3

Figure 5. Tie-Line Flow Metric............................................................................................. 5.4

Figure 6. Response to tripping two units at Palo Verde for several values of gain. Gain, $K_{p}$,

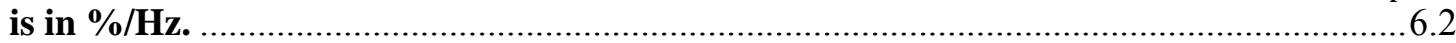

Figure 7. M1, M2, M3 and Time to M1 for a Range of Gains ......................................... 6.3

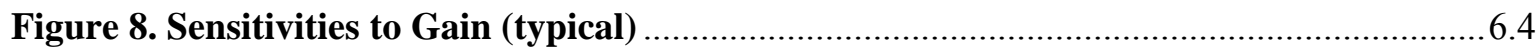

Figure 9. Instability Caused by Delay at two locations for $K_{P}=25 \% / \mathbf{H z}$, Delay $=1 \mathrm{~s} \ldots \ldots \ldots .6 .5$

Figure 10. Metric M3 with 25\% Gain for 2PV Trip in 09HSP Case.................................6.6

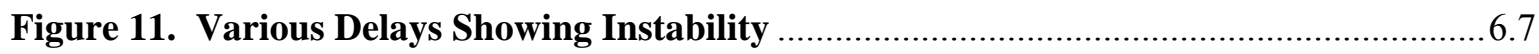

Figure 12. Comparison of Baseline to Demand Response with $\mathrm{Kp}=\mathbf{2 5 \%}$, Delay $=1 \mathrm{~s} \ldots \ldots \ldots .7$

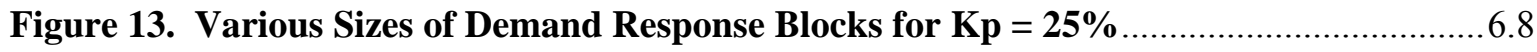

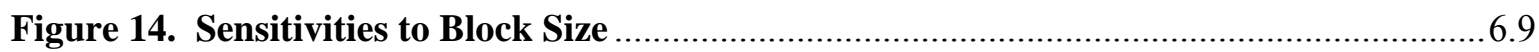

Figure 15. Effect of Localization on Frequency Response .............................................. 6.10

Figure 16. COI Flow for Arizona Implementation versus Alberta Implementation ............6.11

Figure 17. Control Action for Inhibit Function Using 2009HW Base Case..........................6.12

Figure 18. Effect of Inhibit of Restoration Function Showing Frequency Overshoot ..........6.12

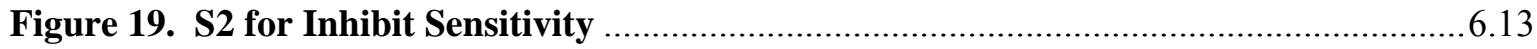




\section{Tables}

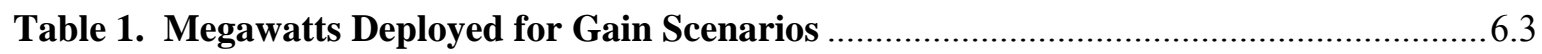

Table A.1. Gain Metrics for 2PV on 2009HSP Case ......................................................... A. 1

Table A.2. Delay Metrics for 2PV on 2009HSP Case......................................................... 2

Table A.3. Block Size Metrics for 2PV on 2009HSP Case ................................................ A.3

Table A.4. Gain Metrics with Inhibit Enabled for 2PV on 2009HSP Case ......................... A.4 


\subsection{Introduction}

Previous studies have shown the potential benefits, as well as potential pitfalls, of implementing autonomous demand response programs [5-8]. None of these studies, however, have offered a comprehensive assessment of the benefits and threats of autonomous demand response on a large-scale model of an interconnected power grid. This research attempts to bridge the gap between the previous studies on research-scale power system models and the full-scale, realistic models used in industry. The study further attempts to validate the benefits, to set boundaries on the known threats, and to possibly identify additional vulnerabilities or implementation issues.

The study uses three 2009 Western Electricity Coordinating Council (WECC) base cases to conduct "loss-of-generation" studies that incorporate autonomous demand response. Loss of generation is the most common contingency that creates a rapid and significant imbalance between instantaneous supply and demand within a power system. A supply/demand imbalance causes an excursion from the nominal or steady-state system frequency. The scope of this study is limited to frequency response, although Trudnowski et al. [5] shows that frequency response is not the only potential beneficiary of autonomous demand response.

Four loss-of-generation contingencies were established for each of the three WECC base cases, resulting in 12 study conditions. For each study condition, approximately 500 simulations were conducted to study various aspects of autonomous demand response. The duration of the simulation was 45 seconds so that primary and secondary frequency control features could be captured and analyzed - at least to the extent they are accurately modeled in the base case. Results from the more than 6000 simulations were processed and summarized to generate "sensitivities" to each of five characteristics of a generic autonomous demand response controller.

A generic demand response controller was developed and implemented in the EPCL programming language. EPCL is a programming language associated with user-defined modeling in the Positive Sequence Load Flow (PSLF) power system study software suite available from GE Energy [9]. One intended outcome of the study was to promote the further study of autonomous load control by the engineering community at-large, and it is hoped that the development and dissemination of the generic control model will facilitate this goal. See Appendix F for a sample listing of the EPCL code used in these studies.

The research seeks to develop sensitivities of autonomous demand response to various controller parameters, and the scope is limited to frequency response. Therefore, several metrics related to frequency response were developed and analyzed by varying the relevant controller parameters. The parameters of interest were:

- Gain: How does varying controller gain affect system frequency response?

- Delay: What effects do various sensor/actuator delays have on system frequency response?

- Block Size: If the control action were discretized, as in widespread use of under-frequency load shedding applied to distribution feeders, what effect would this discretization have on system frequency response? 
- Localization: If the autonomous demand response was constrained to one geographical locale, what effect would this have on system frequency response?

- Inhibiting Restoration: Some devices, such as the compressor unit of an air conditioner, cannot be restored to its loaded condition for several minutes after it has been disconnected. What effect would this "inhibit restore" characteristic have on system frequency response in a system incorporating autonomous demand response?

The study represents a comprehensive examination, on a realistic system model, of the use of autonomous demand response as applied to power system frequency response. It is hoped that the results of this study will generate further discussion, study, and action in this area. 


\subsection{Background}

Demand response has been an integral part of power system control and operation since the early days of electricity use. This practice continues today, with most major utilities in North America still pursuing some form of demand response. ${ }^{1}$ Recently, demand response has received more intense scrutiny as a potentially effective tool to help gain higher levels of asset utilization on the bulk power grid and to avoid or delay the need for construction of new transmission lines [11, 12].

Recent investigations into the use of demand-side resources for enhancing grid operations have been hampered by the artificial limitations in the definitions of the term. For example, the Federal Energy Regulatory Commission (FERC) defines, demand response as "... a reduction in the consumption of electric energy by customers from their expected consumption in response to an increase in the price of electric energy or to incentive payments designed to induce lower consumption of electric energy...." [2]. Similarly, Illian [13] defines demand response as "... a tariff or program established to motivate changes in electric use by end-use customers in response to changes in the price of electricity over time, or to give incentive payments designed to induce lower electricity use at times of high market prices or when grid reliability is jeopardized."

Both FERC [2] and Illian [13] further state that demand response can be implemented either by price signals delivered to the end user or by master control signals sent to the end user. These definitions find no room for autonomous demand response, as described either in this study or previously in [5-7, 10].

Autonomous demand response occurs when load responds to system-based signals rather than master control signals from a control center. The most readily available system-based signal available to all system loads is system frequency, which can be a very reliable indicator of grid instability, problems, or abnormal conditions. In fact, system frequency is the primary signal used today by central generating plants to implement primary frequency control ${ }^{2}$ and to maintain a continuous balance between supply and demand. Allowing load to provide primary frequency control should not be a major extension to those engineers who are well versed in the use of speed governors to control generators. In essence, the concept is to add a speed governor to many system loads.

The benefits of autonomous demand response have been shown in the literature [5-7]. These studies were conducted on reduced-order study systems and focused primarily on determining safe penetration levels. We know of no studies that have comprehensively assessed the technology on a realistic base case, such as has been done in this study.

\footnotetext{
${ }^{1}$ See, for example, PJM Interconnection [10].

${ }^{2}$ See definitions, page v.
} 


\subsection{Power System Model and Modeling Assumptions}

In this study, a set of base cases developed by WECC and its member utilities was used to study the power grid of the western United States and Canada for three system loading and topology configurations throughout 2009. Base cases are generally developed as a planning tool to study future power system operations and potential problems. A base case acts as a starting point for "what-if" scenarios. It takes into account the presence or absence of various facilities and devices caused by planned maintenance outages on various facilities, planned construction activities, planned load profiles and load growth, as well as other considerations.

The WECC 2009 base cases, developed prior to 2009, were intended to allow planners from utilities throughout the western power grid to study and make recommendations to adapt to various potential operating conditions. Three base cases were used for this study: 1) a "heavy spring" (HSP) case with significant generation from the hydroelectric resources in the Pacific Northwest; 2) a "heavy winter" (HW) case with significant heating loads in the cold regions of the planning domain; and 3) a "light summer" (LS) case in which maintaining an acceptable voltage profile across all parts of the grid could be a challenge.

The WECC 2009HSP base case represents approximately $130 \mathrm{GW}$ of generation/load, with loads modeled at over 8000 buses. Some of these loads were considered inappropriate for use in this autonomous demand response study. For example, loads in close proximity to direct current (d.c.) converter stations or major generation facilities may not represent controllable load but rather "house" loads for the station in question. For this study, approximately 7990 loads were independently controlled in cases for which determining the effects of localization was not the analytical goal. For cases in which localization was the focus of the analysis, significantly fewer load buses were controlled.

Similarly, approximately 7900 loads totaling 106 GW for the WECC $2009 \mathrm{HW}$ base case, and 8080 loads totaling $90 \mathrm{GW}$ for the 2009LS base case were independently controlled for the purpose of these studies. As will be discussed in more detail in the next section, the "gain" of the controller was defined in units of $\% / \mathrm{Hz}$ at each load bus. Therefore, as an example, a simulation of the 2009LS base case using all available loads with autonomous demand response global controller gain set at $10 \% / \mathrm{Hz}$ would deliver an aggregate 90-MW response for a system frequency deviation of $0.01 \mathrm{~Hz}$.

Figure 1 shows an overview of the transfer capabilities between various geographic regions of the WECC [12]. The regional transfer capabilities are represented at a very high level; the flows depicted do not represent physical transmission lines. The base case used for the study models almost 14,000 branch sections with more detail than can be easily reduced to graphical display. 


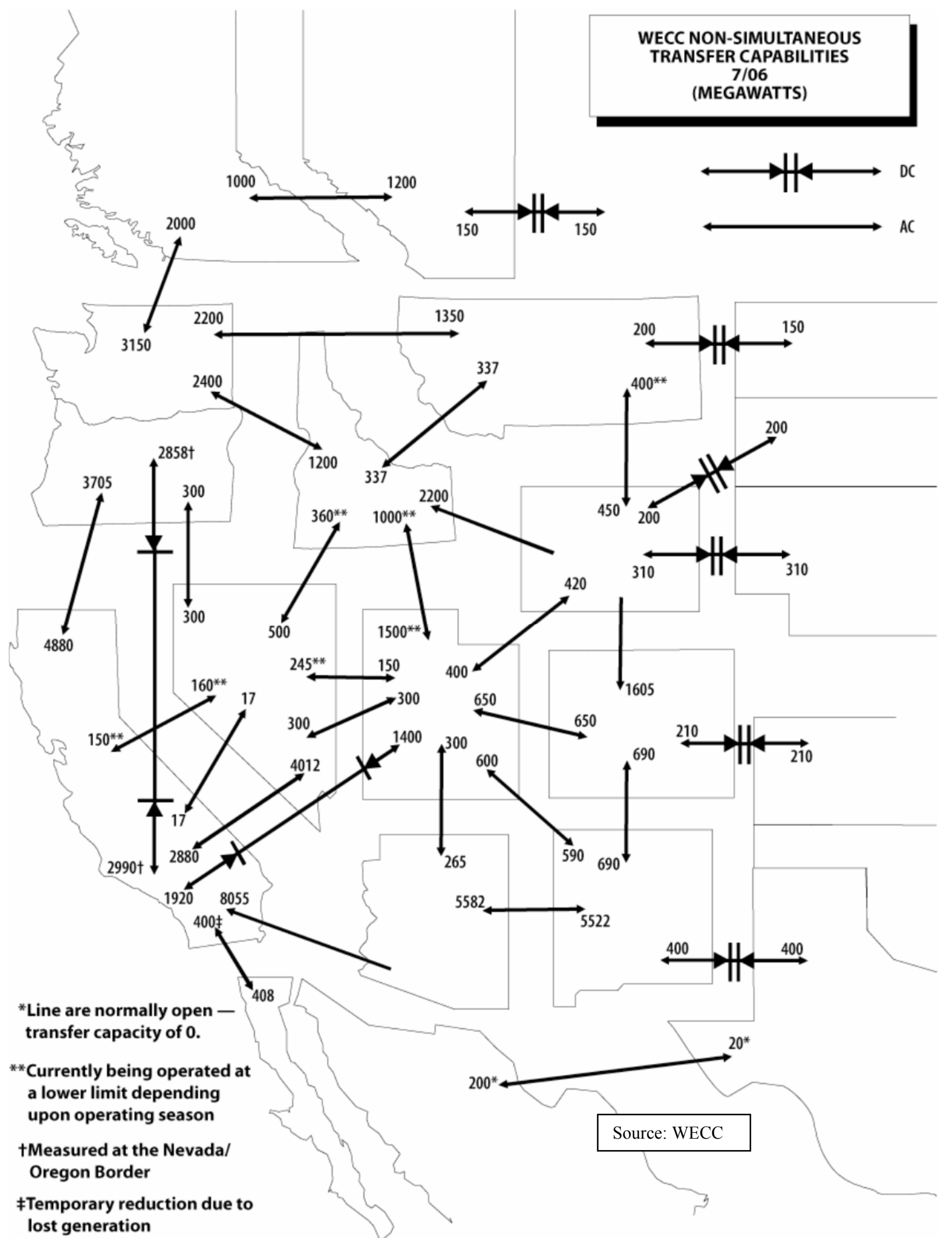

Figure 1. Transfer Capabilities between Various Regions of the WECC 
Using a base case as a starting point, an engineer can simulate various system contingencies such as generator or line outages, load profile change, or equipment failures. For each base case used in this study, four "loss-of-generation" contingencies were simulated. The contingencies were chosen to illustrate both realistic system contingencies and to offer valid insights into localization effects of autonomous demand response.

"Localization" is a term used in this study to address a common and valid issue regarding autonomous demand response programs. A utility in a specific geographic region may decide to implement an autonomous demand response program, but the program is not duplicated throughout the WECC. This scenario might result in a high concentration of autonomous demand response in one geographic region, little or no autonomous demand response elsewhere in the system, and possibly degrade system reliability.

Four regions of interest within the WECC were chosen and loss-of-generation contingencies were developed for each. The following two criteria were used to develop the contingencies:

1. The contingency should result in the loss of approximately $2800 \mathrm{MW}$ of generation.

2. The power system should remain stable for the base case - the case in which the gain of the autonomous demand response controller is zero. If the power system was found to be unstable in the base case, the loss of generation was reduced until stability was achieved.

For the southwest, a contingency involving Arizona generation was developed. Where possible, the study team chose to drop generation at the Palo Verde Nuclear Generating Station. For the northwest, several units in Alberta, Canada were chosen. For the east, generation at the Jim Bridger Power Station was dropped. Finally, a contingency affecting the central Pacific Coast involving a loss of generation at the San Onofre Nuclear Generating Station was devised. In some cases the units involved in the simulated contingency were located at different power plants. Although it is not probable that multiple units at different stations might be involved in a single contingency, this generalization was considered to be adequate because the study team was trying to develop a regional contingency totaling approximately 2800 MW regardless of whether or not the chosen contingency might be physically realizable. For convenience, the four contingencies are referred to as Palo Verde, San Onofre, Jim Bridger, and Alberta.

The four contingencies in each of three base cases provided 12 study conditions from which to devise the relevant sensitivity studies. 


$$
3.4
$$




\subsection{Controller Design}

The control law was designed for flexibility and usability by independent organizations in future work. Figure 2 shows the controller block diagram. The extent of the controller code is shown within the dotted lines. The network solution, shown outside the dotted lines, is represented by the generic transfer function $G(s)$. The error term also is formed within the network solution.

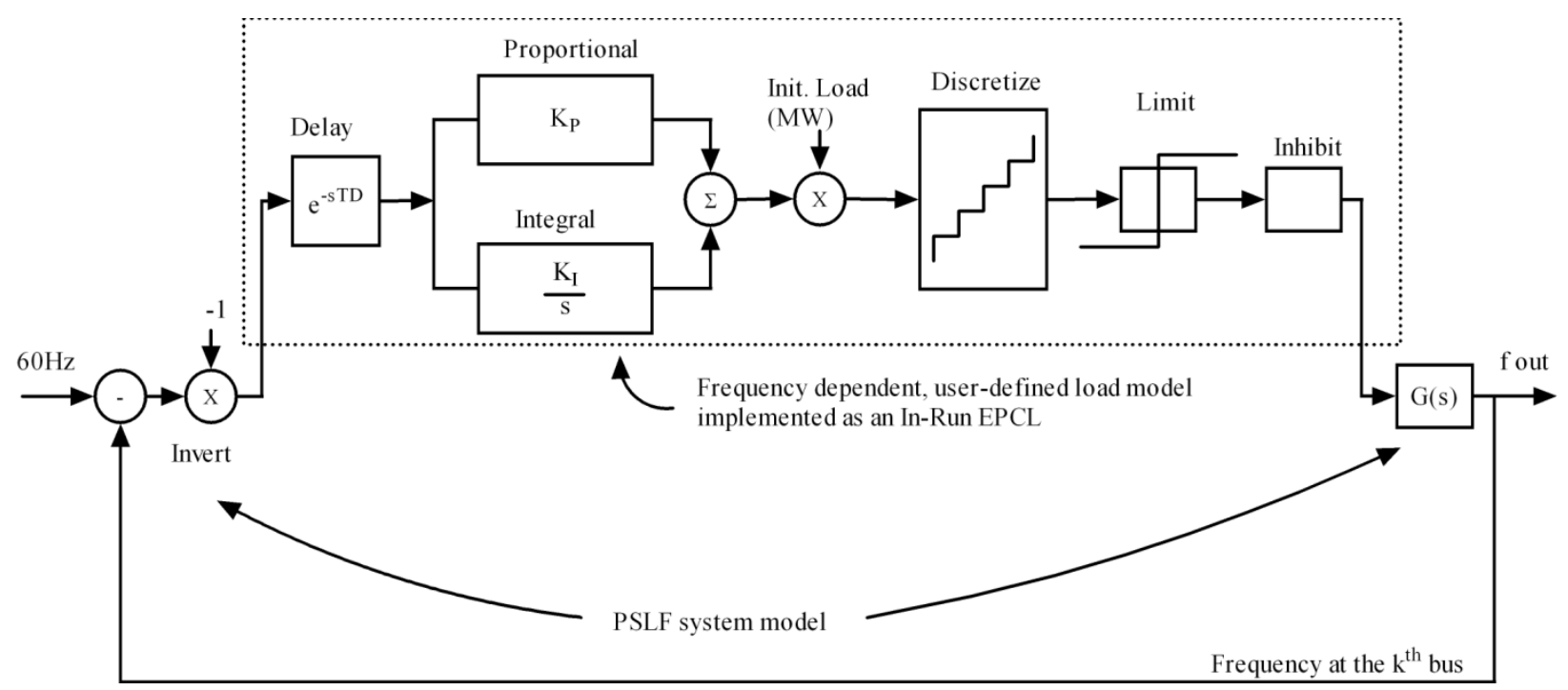

Figure 2. Controller Block Diagram

A description of the intended function of each of the blocks follows. The list flows from the left-most node of the control law to the right side.

- Error: Returns the error between the reference frequency and the bus frequency.

- Invert: Inverts the error so the negative error corresponds to a request to shed load.

- Delay: A "pure" delay block models two system delays - the delay inherent in the device (e.g., a water heater) frequency transducer and the delay inherent in the device's on/off contactor. The frequency transducer detects system frequency and provides a signal to the internal controller of the device. The contactor is the actuator that physically controls the energy consumed by the device.

- Proportional and Integral Control Blocks: Implements the core of the simple control law. $\mathrm{K}_{\mathrm{p}}$ is the proportional controller gain, and $\mathrm{K}_{\mathrm{I}}$ is the integral controller gain. More information on the nature of the gain block is provided below. Note that the value of $\mathrm{K}_{\mathrm{I}}$ was always zero for this portion of the studies.

- Normalize: Normalizes the controller gain to a megawatt value to be controlled at this bus. The gain is most often represented in percent of load at this bus per $\mathrm{Hz}(\% / \mathrm{Hz})$, but also can be represented in MW per $\mathrm{Hz}(\mathrm{MW} / \mathrm{Hz})$.

- Discretize: Models the possibility that some loads will be shed or added in blocks rather than in a continuous control mode with infinite variability. The units are "MW/Step." In other words, if the user specifies $1 \mathrm{MW} / \mathrm{Step}$, the load at a given bus will not change until the overall megawatt response exceeds $1 \mathrm{MW}$. 
- Limit: Models a limit at each bus. Units are percent of initial load at this bus. For example, the modeler can specify that no more than 5 percent of the load at any given bus may respond.

- Inhibit: If set, the load can be shed but cannot return to the "on" state. This simulates equipment, such as an air conditioner, that can be turned off but cannot be turned back on immediately.

Note that the simulation alternates between the network solution and the controller solution. Within the controller portion, the solution iterates through every load bus involved in the study setting the modified value of load at each bus in accordance with the measured frequency at that bus.

To further illustrate this important point, Figure 3 shows frequency as calculated by the simulation tool at two buses, one near a generator that was taken out of service at $t=3 \mathrm{~s}$, and one at some distance from the disturbance. The figure illustrates that each bus in the control law "sees" a unique frequency at any given time in the simulation.

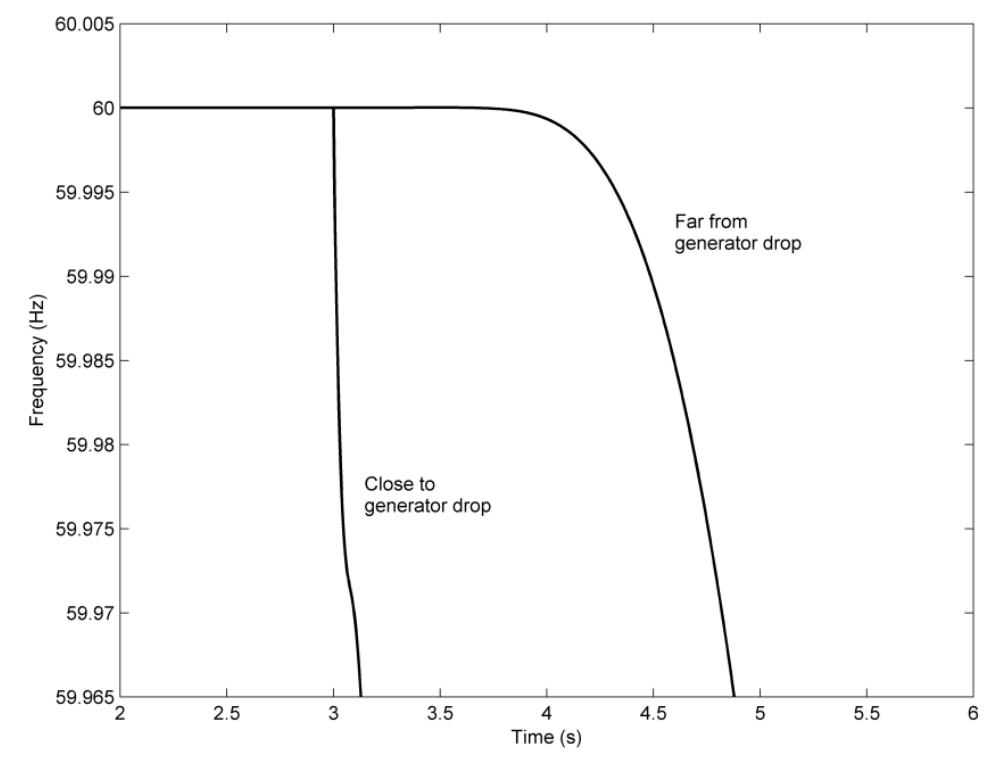

Figure 3. Example of Frequency at Two Different System Locations

Within the controller software code block, and throughout this document, gain is expressed either in units of $\% / \mathrm{Hz}$ or $\mathrm{MW} / \mathrm{Hz}$ depending on the value of an internal flag variable. In most cases we found it much more intuitive to implement the gain in units of $\% / \mathrm{Hz}$, meaning that a proportional gain of 10 percent per Hertz implements control action on 10 percent of the pre-disturbance load at a given bus for a frequency deviation of $1 \mathrm{~Hz}$ at that bus. Similarly, a gain of $10 \% / \mathrm{Hz}$ would yield a response of 1 percent of the pre-disturbance load for a frequency deviation of $0.1 \mathrm{~Hz}$. For all simulations, the pre-disturbance load at the bus is defined to be 100 percent at that bus, so at each time step in the simulation gain calculations uses a constant reference value.

Controller delay, represented by the delay block within Figure 2, is intended to incorporate cumulative real delays in sensing and tripping a low-voltage appliance. The speed with which highvoltage utility equipment senses and interrupts the flow of current is well studied and understood. The 
need for rapid response times also is well understood. At the residential or commercial appliance level, however, rapid response times have not been a significant engineering driver. Incorporating delay into the control law allows broad-based studies of the effect of delays.

The flexible controller design shown in Figure 2 was implemented in the EPCL programming language, which is an appendage of the PSLF power system simulation suite [9]. Within PSLF, the control law is implemented as an In-Run EPCL executing at each time step of the underlying power system simulation. A complete listing of a typical EPCL program used in this study is provided in Appendix F. The approach to conducting the study was then to perturb one of the controller parametersgain, delay, block size, localization, and inhibit — and to measure the system response. The methodology is further described below. 
4.4 


\subsection{Sensitivity Studies}

With comprehensive and flexible power system and controller models available, sensitivity studies were initiated. Our general approach was to simulate a response to 1 of the 12 system conditions using one set of control parameters, and then after slightly modifying one of the parameters, perform another simulation. For this study, a measure of sensitivity was considered to be a change in system response as a result of an incremental change in a control parameter. Five controller parameters were considered for evaluation in this study. They were:

- Gain - How does varying controller gain affect system frequency response?

- Delay - What effects do various sensor/actuator delays have on system frequency response?

- Block size - If the control action were discretized, as in widespread use of under-frequency load shedding applied to distribution feeders, what effect would this discretization have on system frequency response?

- Localization - If the autonomous demand response was to be constrained to one geographical locale, what effect would this have on system frequency response?

- Inhibit of Restoration - Some devices, such as the compressor unit of an air conditioner, cannot be restored to its loaded condition immediately after being disconnected. What effect would this "inhibit restoration" characteristic have on system frequency response in a system incorporating autonomous demand response?

\subsection{Metrics for Evaluating Sensitivities}

Readers should note that the system model is highly nonlinear. Perhaps more importantly, there are no universally accepted definitions of "good" system frequency response. If a sensitivity to one of the five aforementioned controller parameters is to be defined as the change in parameter divided by change in system response, it is imperative that the denominator be quantifiable.

Recent work sponsored by FERC and the U.S, Department of Energy, as well as extensive recent work by North American Electricity Reliability Corporation (NERC) and WECC subcommittees, have provided insights into some of the questions listed above [4, 13-20]. One recent study by the Lawrence Berkeley National Laboratory (LBNL) suggests the following three relevant metrics for evaluating the performance of an interconnected grid with respect to frequency response [4]:

1. Frequency Nadir - This metric is defined simply as the minimum frequency following a loss-ofgeneration event.

2. Nadir-Based Frequency Response - This metric is defined as generation lost/(60 Hz minus the frequency nadir) for this study, given that all simulations start with a pre-disturbance frequency of $60 \mathrm{~Hz}$.

3. Primary Frequency Response - As proposed, this metric is to be evaluated at discrete times following a system disturbance. Primary frequency response was measured by summing the change in generator real power output resulting from primary frequency control (e.g., governor action) for all generators in an area of interest, usually within the area of a balancing authority. 
The metrics developed by LBNL are useful for evaluating classical governor response; however, it remained difficult to accurately apply any metrics to such a diverse set of data as was collected in the course of this study. The frequency nadir, for example, is different at different points in the system. The nadir also can be difficult to define if a generator at a nearby bus is lightly damped and is causing system frequency to oscillate. To overcome these challenges with this metric, the study team chose to calculate the mean of the bus frequency at several points in the system, to then low-pass filter the resultant signal, and to find the minima of the filtered signal. This value was declared to be the frequency nadir for the simulation in question. Using this approach, a consistent measurement for this metric throughout all of the study cases could be generated.

This averaging and filtering did not prove to be successful for all of the metrics in all cases. The study team found that additional metrics would be helpful for studying the interaction of autonomous demand response with classical governor response. It should be noted that, with autonomous demand response, the load performs a portion of the primary frequency control that formerly was done entirely by governor action. Generators are less burdened for frequency regulation and can be operated with lower reserve margins in a system with autonomous demand response. In the extreme, it is conceivable that all primary frequency control could be implemented by the demand side, leaving the supply side to take care of secondary frequency control ${ }^{1}$.

The metrics used to evaluate the sensitivity studies undertaken in this study include two of the LBNL metrics along with several additional metrics that proved useful in some simulations. Figure 4 depicts the basis for some of the proposed metrics. The figure shows the frequency response at a typical bus caused by a loss-of-generation contingency. The solid trace is actual frequency and the dashed trace is the same signal after low-pass filtering with a corner frequency of $0.15 \mathrm{~Hz}$. The following five values are shown in the figure (from left to right):

1. Slope of inertia response, given by $\Delta f / \Delta t$;

2. Frequency nadir, which is about $59.76 \mathrm{~Hz}$ in this example;

3. Time of frequency nadir, which occurs at about $11 \mathrm{~s}$ in this example;

4. Settling frequency, which is about $59.86 \mathrm{~Hz}$ in this example;

5. Time of settling frequency, which occurs at about $26 \mathrm{~s}$ in this example.

\footnotetext{
${ }^{1}$ This concept, partially described in a 1982 patent by Schweppe [21] and followed by more recent work [5-7], show that primary frequency response delivered by the demand side (i.e., the megawatts of control action delivered by the load) can be accurately estimated given knowledge of the control law, the penetration levels of autonomous-acting devices, and measured system frequency.
} 


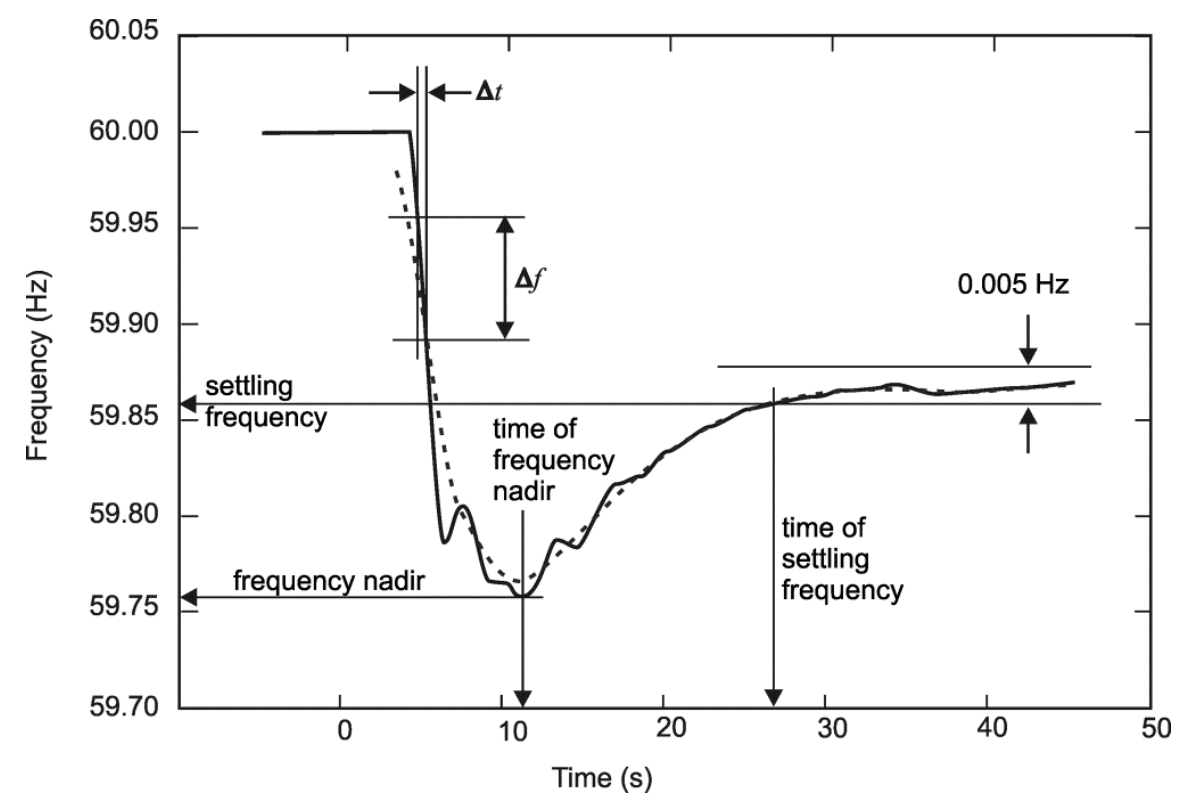

Figure 4. Basis for Frequency Response Metrics

The slope is evaluated on the raw, unfiltered signal in an interval beginning $0.5 \mathrm{~s}$ after the contingency and ending $2.5 \mathrm{~s}$ after the contingency. The primary purpose of this metric is to evaluate what impact responsive loads might have on the inertia response of the system.

The frequency nadir is evaluated using the filtered signal. It is a simple lookup of the minimum frequency (or maximum in the case of a load shedding event) encountered during the simulation.

The settling frequency also is evaluated using the filtered signal. The filtered signal is continuously assessed for "stability" by evaluating whether the signal stays within a frequency band of $\pm 0.005 \mathrm{~Hz}$ of the frequency at $t=45 \mathrm{~s}$, which is the end time of the simulation. As soon as the algorithm determines that the frequency is stable, it returns the first data point that satisfies the criteria. We define this point as the "settling point."

With respect to appropriate metrics for the sensitivity studies, we believed it would be helpful to provide a measure of settling frequency as well as a measure of interchange imbalance in addition to some of the metrics proposed in [4]. Figure 5 shows a different basis used to evaluate deviations in interchange flow. The figure depicts the sum of megawatt flows on all the lines comprising a designated path of interest. 


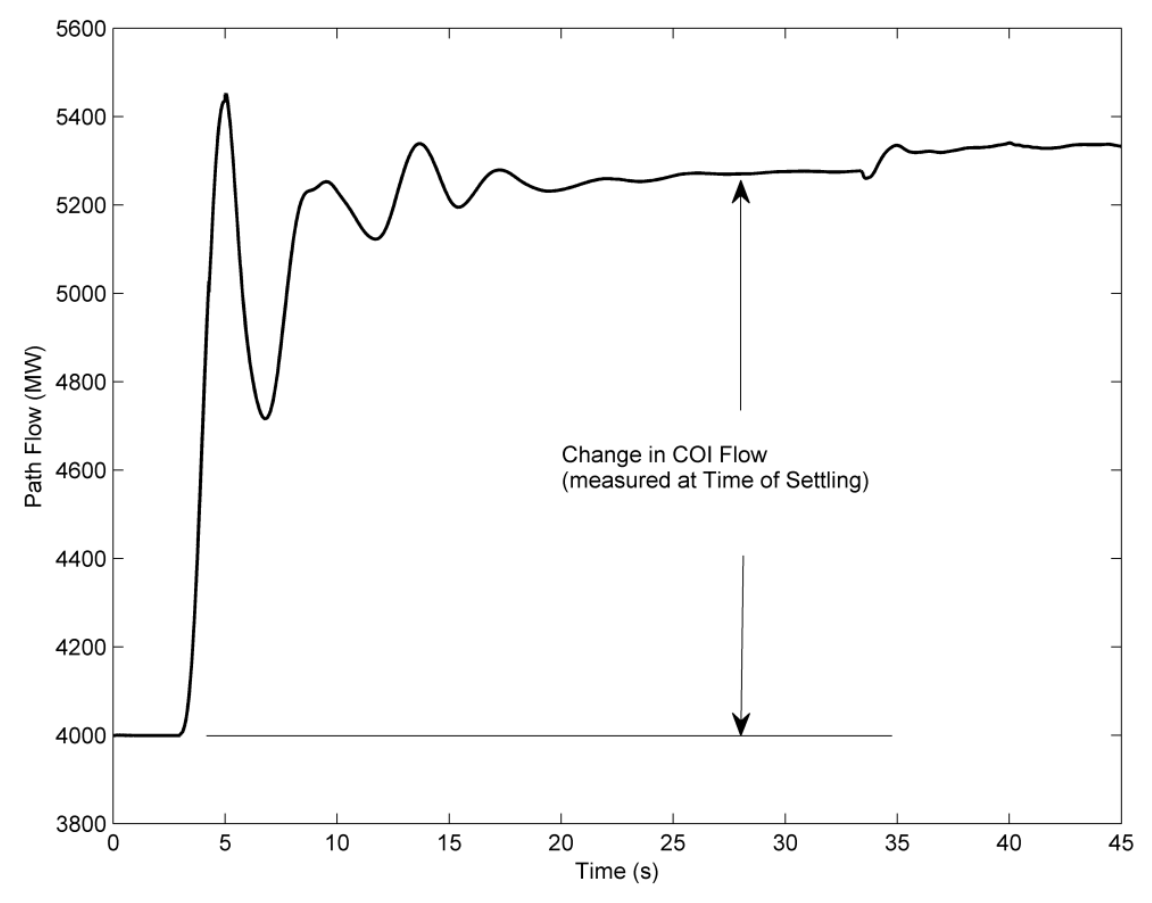

Figure 5. Tie-Line Flow Metric

The relevant value is the difference between tie-line flow before the contingency and tie-line flow at the settling time (i.e., the time at which the frequency reaches its settled value).

Given the preceding discussion, five metrics, identified as M1 through M5, were explicitly defined for purposes of evaluating the sensitivities, identified as S1 through S5, of various autonomous demand response controller parameters. For example, S1 might define the sensitivity to M1 for deviations in the gain parameter of the controller on a particular set of simulations. The six metrics are:

1. Frequency Nadir (M1) - We used the same definition for this metric as LBNL, but we evaluated it by averaging and filtering frequencies at six buses within the system. The six buses used in the average are 1) Camino, 2) Coulee, 3) Williston (Alberta), 4) Colstrip, 5) Navajo, and 6) Lugo.

2. Settling Frequency (M2) - We evaluated this metric by averaging and filtering the frequencies at the same six buses as defined above in M1. We then found the first data point following the disturbance and recovery meeting the criterion that all points after this point must have a deviation less than 0.005 $\mathrm{Hz}$ from the final frequency value $($ at $\mathrm{t}=45 \mathrm{~s})$.

3. Time of Settling (M3) - We defined this metric as the time of the point defined in M2 minus the time of the occurrence of the contingency.

4. Nadir-Based Frequency Response (M4) - For this metric, the same definition as described in Eto et al. [4] was used but the frequency signal was averaged and filtered as in M1. The metric is generation lost divided by $(60-\mathrm{M} 1)^{1}$. The amount of lost generation used in the numerator was determined from the pre-contingency base case.

\footnotetext{
${ }^{1}$ While the metric, as defined in Trudnowski et al. [5], is generation lost divided by initial frequency minus frequency nadir, the initial frequency in simulation is always $60 \mathrm{~Hz}$.
} 
5. Normalized Change in California-Oregon Interchange (COI) Flow (M5) - We defined this metric as the change in COI flow divided by the amount of generation lost from pre-disturbance values to the time defined in M3. COI flow was used because it is very relevant in the WECC, and because it was difficult to find a meaningful metric that incorporated tie-line flow averages from several regions. Tie-line flow is a relevant metric because load will respond differently than generation in response to a loss-of-generation event. Localization of autonomous demand response can have a significant impact on deviations from scheduled interchange in the aftermath of a system event.

These five metrics were determined to be sufficient to produce meaningful results for our sensitivity studies. Before ending this discussion of how to measure performance, it is prudent to address the meaning of "beneficial" to system reliability and what might be meant by a "threat" to system reliability in terms of each of the proposed metrics.

1. M1 is the low point of the system frequency response. A "good" value would be close to $60 \mathrm{~Hz}$. If a control action increases the value of this metric in response to a loss-of-generation event, it would be considered a benefit. Lower values in response to a control action would be considered a threat to system reliability.

2. M2 is the final value of primary frequency control. A "good" value would be close to $60 \mathrm{~Hz}$. If a control action increases the value of this metric in response to a loss-of-generation event, it would be considered a benefit. Lower values in response to a control action would be considered a threat to system reliability.

3. M3, the settling time, represents the speed with which primary frequency control acts. A "good" value would be close to the time of the initial disturbance, in this case $3 \mathrm{~s}$. If a control action decreases the value of this metric in response to a loss-of-generation event, it would be considered a benefit. Higher values in response to a control action would be considered a threat to system reliability.

4. M4 is similar to M1, but the frequency deviation is scaled to the magnitude of the initial disturbance. A "good" value would be very high. Perfection would be a value of infinity, which would mean that the combined generation and load controllers would respond instantaneously and with great strength to even the slightest deviation in system frequency. Of course, this effect may not be desirable.

Nevertheless, a high value of M4 to a loss-of-generation event would be considered a benefit in this context. Lower values in response to a control action would be considered a threat to system reliability.

5. M5, the change in COI flow, describes a deviation between scheduled and actual tie-line flow. If autonomous demand response were to perfectly compensate for a loss-of-generation event within the bounds of a given balancing authority, then all tie-line schedules would be maintained. By this reasoning, a "good" value for M5 would be close to $0 \mathrm{MW}$. If a control action decreases the value of this metric in response to a loss-of-generation event, it would be considered a benefit. Higher values in response to a control action would be considered a threat to system reliability. 


\subsection{Discussion}

This section summarizes the results of the simulations performed during the course of the project. The metrics described in the previous section, and the sensitivities to these metrics, are presented. Some of the metrics proposed did not prove to be valuable assessment tools for some of the studies. For example, the research showed that large delays can cause severe stability problems. In these cases, the system never settled to a steady-state condition. Because the physics of small signal stability is quite different from that of frequency response, the metrics developed to measure frequency response are not adequate. Where this and similar issues occur, they are duly noted.

The study results are grouped according to the respective analysis categories. Additional details and raw data for each of the analysis categories can be found in the appendices.

\subsection{Sensitivity to Gain}

An artifact of the controller design used for these studies is that there is no distinction between the gain of an individual controller and the overall penetration level of autonomous demand response controllers within the system. At any given load bus, a controller gain of 10\%/Hz might mean that 10 percent of all loads at this bus are being controlled with a gain of $100 \% / \mathrm{Hz}$ or that the controllers of all loads at this bus have a gain of $10 \% / \mathrm{Hz}$.

This feature of the control law creates no loss of generality. Recall that the units of gain are $\% / \mathrm{Hz}$, where "\%" means the percent of pre-disturbance load at this bus. Therefore, a gain of $10 \% / \mathrm{Hz}$, means that 10 percent of the load at this bus responds for a frequency deviation of $1 \mathrm{~Hz}$. It has been suggested that actual implementation of the control law be distributed and that the control law have a stochastic component with respect to the frequency trigger point [5]. With this approach, an individual device can be considered to have a gain of 100 percent for a discrete frequency trigger point. An aggregated group of identical devices with frequency trigger points selected randomly, but conforming to a uniform probabilistic distribution from 60 to $59 \mathrm{~Hz}$, can be considered to have a gain of $100 \% / \mathrm{Hz}$. Similarly, if the probabilistic distribution was not uniform and/or if the distribution did not have a range of $1 \mathrm{~Hz}, \mathrm{a}$ different equivalent aggregated gain could be calculated.

Consider a group of electric water heaters, each with a rating of $4 \mathrm{~kW}$. The water heaters are either on or off (i.e., consuming $4 \mathrm{~kW}$ or consuming $0 \mathrm{~kW}$ ). Now consider a situation in which a group of 100 of these water heaters were fitted with autonomous demand response controllers with discrete frequency triggers such that the water heater was forced to the "off" state if frequency dropped below the trigger value, and that each of the water heaters had a different frequency trigger set point spaced at equidistant $0.01 \mathrm{~Hz}$ intervals declining from $60 \mathrm{~Hz}$. There would therefore be 100 water heaters, with uniformly spaced set points between 60 and $59 \mathrm{~Hz}$. Assuming these water heaters were all initially in the "on" state, this aggregated group of water heaters could be modeled as a single load having a gain of $400 \mathrm{~kW} / \mathrm{Hz}$.

The hypothetical load configuration described above is discussed more generally in Trudnowski et al. [5]. Further, the study of probabilistic modeling of aggregated loads under decentralized, coordinated control is currently being investigated by others [22]. Although the example describes appliance-sized loads, the control theory could be generalized to loads in commercial and industrial facilities, e.g. variable-speed drives. The key point is that aggregated load can be reasonably modeled, under the 
assumption that the control law for each load in the aggregation is coordinated by means of, for example, a published industry standard, such that the study of the model's frequency response is valid.

It is reasonable to further assume that the host utility may have only crude methods for controlling the level of penetration of autonomous demand response devices on their system. Suppose, for example, that the utility sponsored a cash-back rebate program for purchasers of water heaters fitted with autonomous demand response controllers. If, as is asserted herein, penetration level is equivalent to increased gain, several issues would have to be addressed: the timing of stopping the rebate program, danger to system stability resulting from excessive gain, determining how much gain would give sufficient results, etc. The project team tried to address these issues through simulations.

Figure 6 shows a set of traces of frequencies near Grand Coulee Dam in central Washington State in response to tripping two units at Palo Verde. The base case for this simulation was the $2009 \mathrm{HW}$ case, but we found similar results for all base cases. It was very difficult to find any detrimental effect to high gain, or high penetration levels, in the absence of other factors, such as excessive delay.

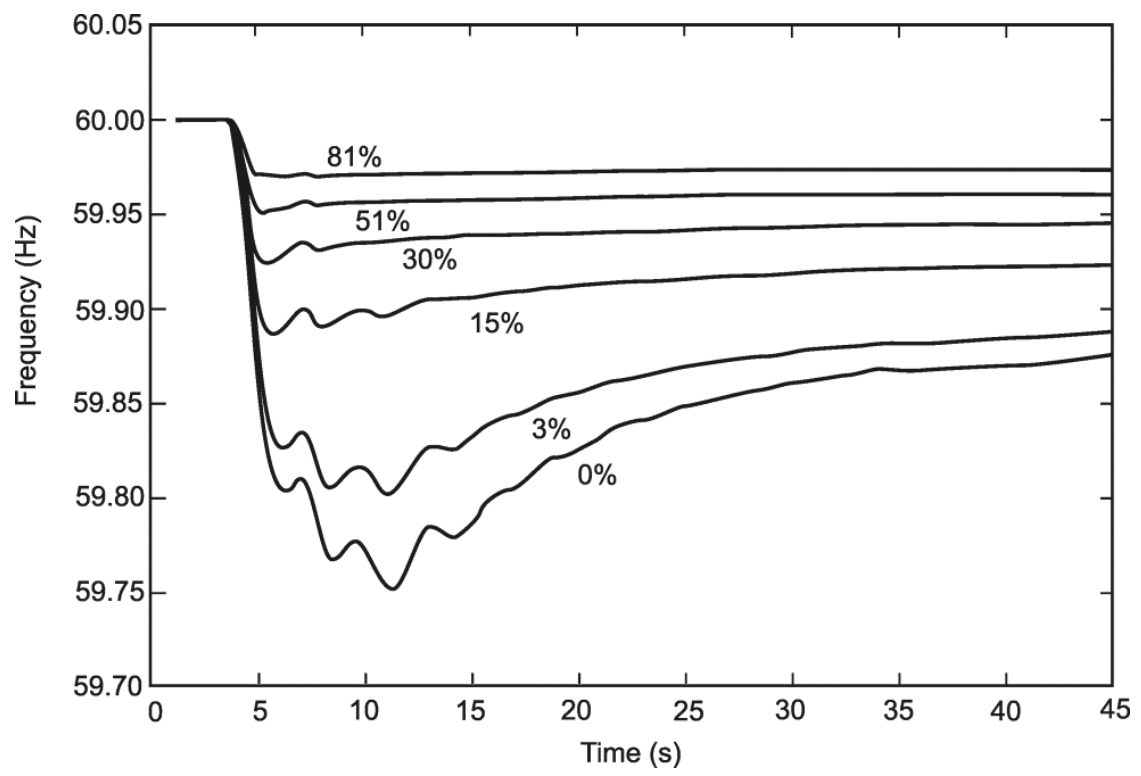

Figure 6. Response to tripping two units at Palo Verde for several values of gain. Gain, $K_{p}$, is in $\% / \mathrm{Hz}$.

Note that there is a significant improvement in system performance with only $15 \%$ gain, and that beyond approximately $30 \% / \mathrm{Hz}$, there appears to be a decreasing marginal rate of return, as measured by improvement in M1 (frequency nadir).

Further, it is significant to note that at some level of gain the metric M1 and the metric M2 (settling frequency) are indistinguishable. This situation is observable in Figure 6 at a gain of $81 \% / \mathrm{Hz}$, and it is noteworthy because the sensitivities to these metrics become meaningless at this point.

Table 1 shows that M3 (time to settling frequency) changes significantly with gain. The data in Table 1, which are related to the results depicted in Figure $6^{1}$, show the megawatts of autonomous load response deployed for each of the gain levels shown in the figure.

\footnotetext{
${ }^{1}$ The table shows filtered values, while the figure shows unfiltered time series information.
} 
Table 1. Megawatts Deployed for Gain Scenarios

\begin{tabular}{|r|r|r|r|r|r|r|}
\hline Gain & MW Deployed & M1 & M2 & M3 & M4 & M5 \\
\hline 0.0 & 0.0 & 59.77 & 59.87 & 28.9 & 12008.9 & 1273.7 \\
\hline 3.0 & -618.5 & 59.81 & 59.88 & 29.1 & 14791.7 & 1194.6 \\
\hline 6.0 & -1038.0 & 59.84 & 59.89 & 27.9 & 17696.3 & 1133.1 \\
\hline 9.0 & -1369.5 & 59.86 & 59.90 & 26.2 & 20566.8 & 1082.9 \\
\hline 12.0 & -1623.2 & 59.88 & 59.91 & 25.6 & 23390.2 & 1045.4 \\
\hline 15.0 & -1819.6 & 59.89 & 59.92 & 23.7 & 26220.2 & 1011.5 \\
\hline 30.0 & -2311.0 & 59.93 & 59.94 & 15.6 & 41387.7 & 906.4 \\
\hline 51.0 & -2607.0 & 59.96 & 59.96 & 13.1 & 62792.9 & 849.5 \\
\hline 66.0 & -2697.3 & 59.96 & 59.97 & 13.1 & 78372.1 & 831.0 \\
\hline 81.0 & -2750.3 & 59.97 & 59.97 & 13.1 & 94057.6 & 818.4 \\
\hline
\end{tabular}

To further illustrate the finding that there are diminishing returns to increasing gain, Figure 7 shows graphically the metrics M1, M2, and M3 for a range of gains on a typical base case and contingency.
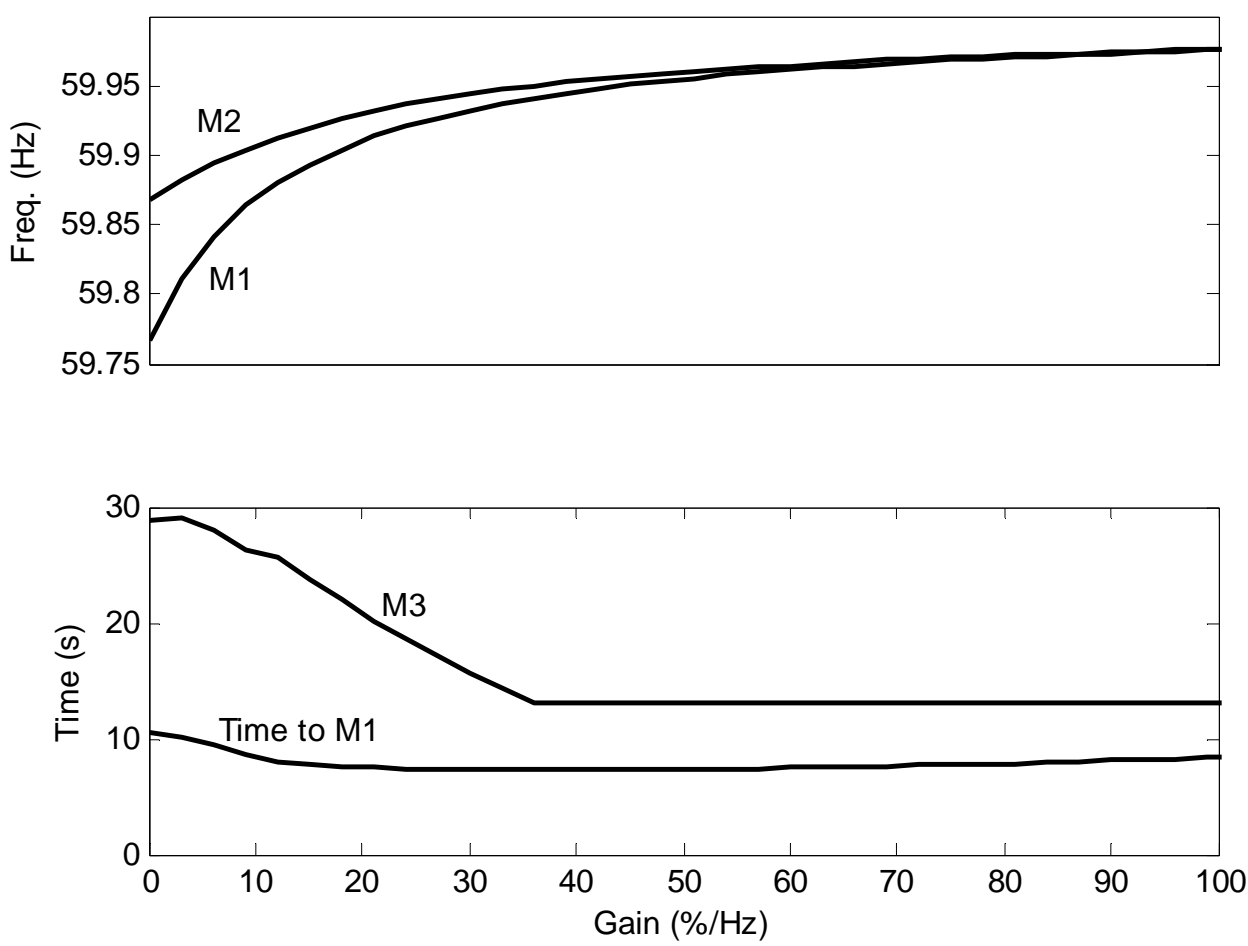

Figure 7. M1, M2, M3 and Time to M1 for a Range of Gains

Figure 8 shows a set of typical sensitivities generated from this study. From top to bottom, the figure shows the sensitivities of metrics M1 through M5. The respective sensitivities are labeled S1 through S5. The ordinate for each data point represents the change in the underlying metric for a unit change in gain. For example, the units of S1 would be $\Delta \mathrm{Hz} / \Delta \mathrm{Gain}$. We can see from $\mathrm{S} 1$ that at some low value of gain, approximately $6 \% / \mathrm{Hz}, \mathrm{S} 1$ is approximately 0.01 . Therefore, in the vicinity of $\mathrm{K}_{\mathrm{p}}=6 \% / \mathrm{Hz}$, an incremental increase in gain of $1 \%$, would yield an improvement in M1 of $0.0001 \mathrm{~Hz}$. 

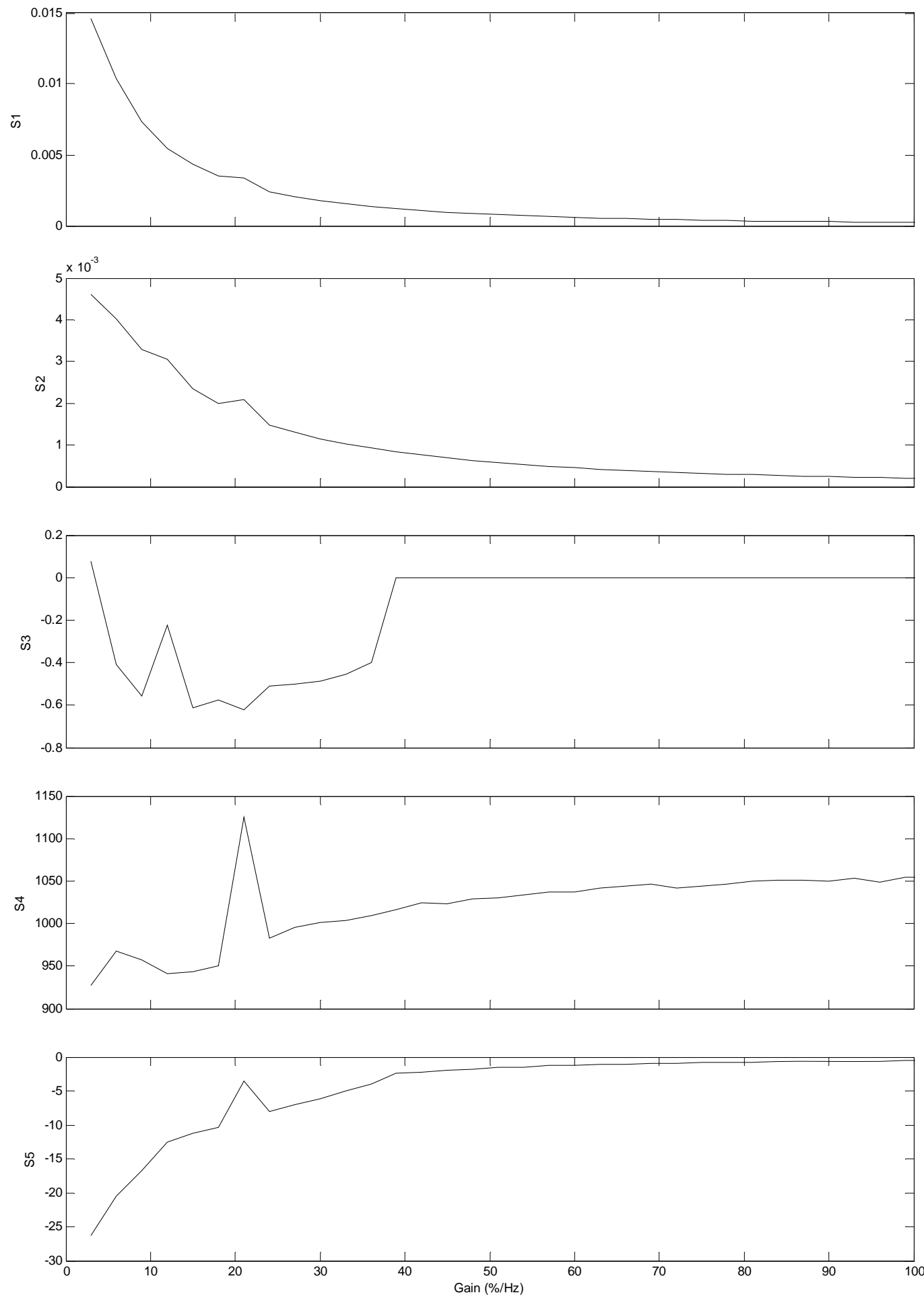

Figure 8. Sensitivities to Gain (typical)

The most significant finding with respect to gain studies in the 12 study conditions was that there were no instances in which increasing gain, while holding the other controller parameters constant, resulted in a condition that adversely impacted the frequency response of the system. It is possible that, with further work, regions of optimal sensitivity might be found that may allow sponsor utilities to decide 
where they might find the greatest value from deployment of autonomous demand response. This analysis was left for the future.

\subsection{Sensitivity to Delay}

While the studies showed little or no detrimental impact on reliability for autonomous demand response at any level of gain (i.e., penetration) for controllers with no inherent delay, there were significant reliability issues related to delays.

An interconnected power system is a dynamic machine, and one facet of system dynamics relates to electromechanical oscillations. In this section, we show that, as the controller delay increases, the autonomous demand response controller begins to inject accelerating power into the system in anti-phase with the electromechanical energy of the system generators. As the delay puts the controller action closer to 180 degrees out of phase with an electromechanical mode of oscillation, the system becomes unstable. Note that it is difficult, if not impossible, to assess metrics designed for the purpose of measuring frequency response when examining an unstable system. For this reason, the frequency response metrics were only evaluated for small values of delay. Figure 9 depicts an extreme example of an unstable condition in which frequency response metrics are not useful.

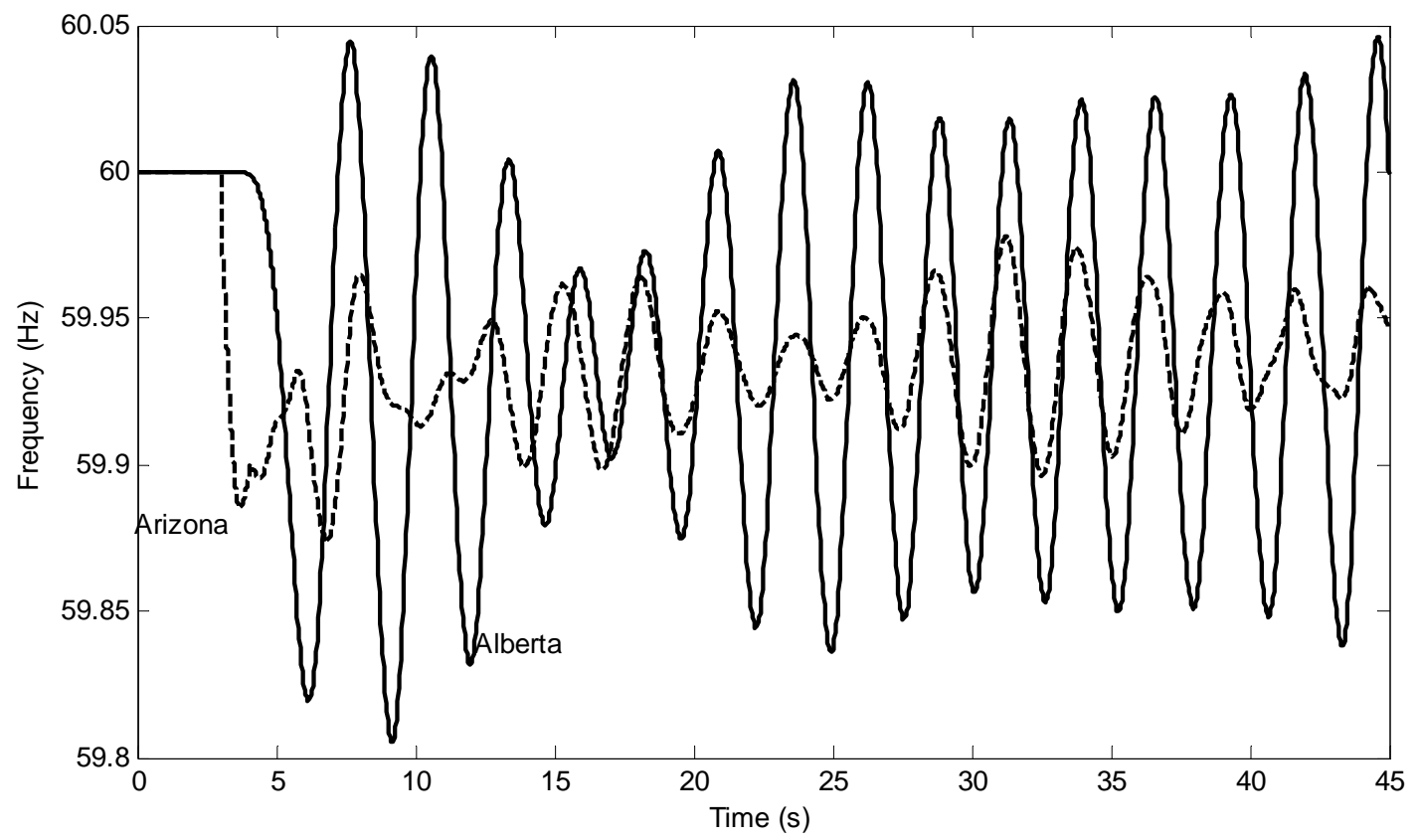

Figure 9. Instability Caused by Delay at two locations for $K_{P}=25 \% / H z$, Delay $=1 \mathrm{~s}$

In this example, the controller is not receiving frequency measurements and/or is not able to act on the frequency measurements until a delay of $1 \mathrm{~s}$ has elapsed. In other words, the controller is acting on system conditions that were occurring 1 second in the past, not on current conditions. This delay is seen to be creating or stimulating one or more modes of oscillation somewhere on the system.

The project team set out to develop a better understanding of what delay values would be safe from the perspective of system stability. In keeping with the primary objective of the research, which is 
frequency response, the exploration into stability regions was not exhaustive but served to yield information that may be useful to the reader. Figure 10 shows metric M3 (time to reach settling frequency) for a set of delay values with gain held constant at $25 \% / \mathrm{Hz}$. This metric was found to be the most suitable for assessing what value of delay might be considered unsafe with respect to oscillatory instability.

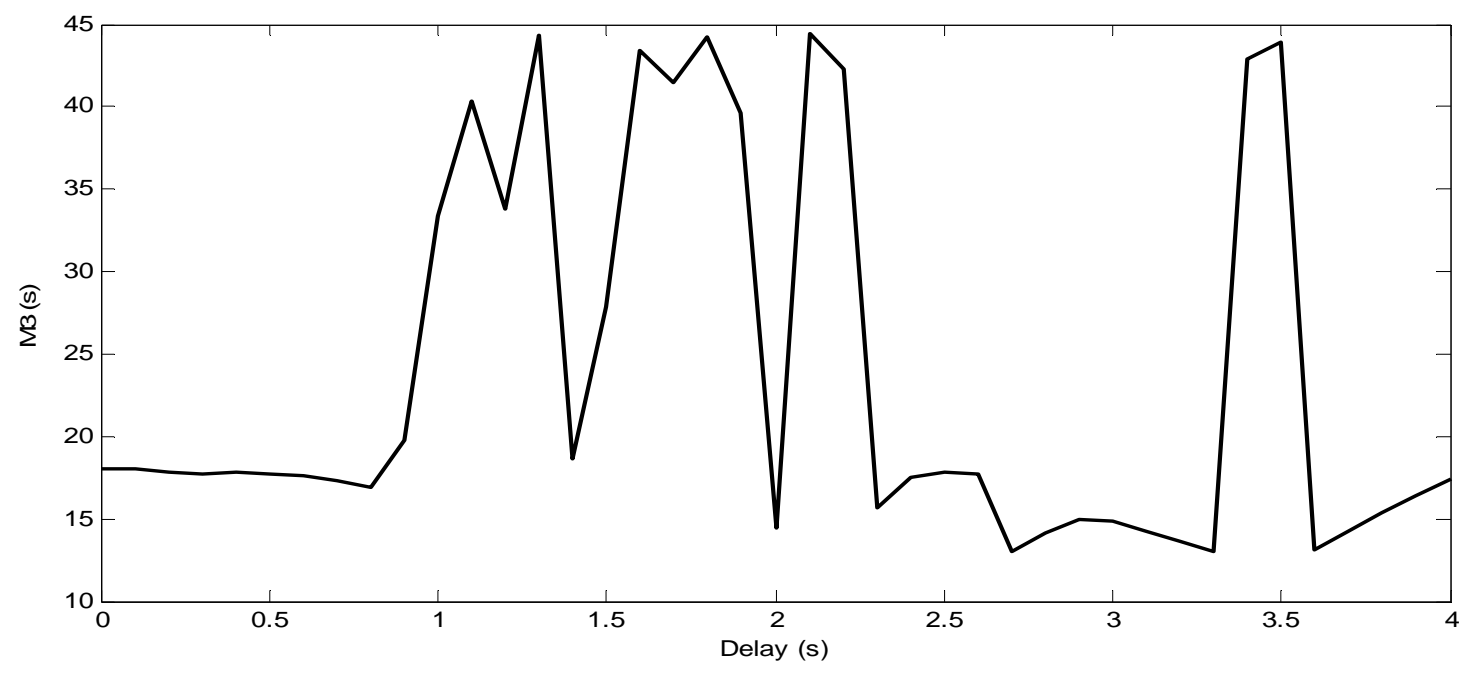

Figure 10. Metric M3 with 25\% Gain for 2PV Trip in 09HSP Case

To begin an interpretation of the figure, recall that the first point on the curve, corresponding to no delay, is the same as the case for which the gain is $25 \% / \mathrm{Hz}$ with no delay. The figure shows that as the delay is increased, M3 changes little until reaching a value of approximately $0.6 \mathrm{~s}$. As a delay value of $1 \mathrm{~s}$ is approached, the settling time abruptly takes on large values. Extremely high values for M3 are incongruous for the purpose of evaluating frequency response. It simply means that the system takes an exceedingly long time to settle to a stable frequency. In this case the metric becomes irrelevant for frequency response and is instead providing some measure of the system's oscillatory instability.

Figure 11 shows time-series plots of frequency at Grand Coulee in response to the loss of generation event for various delay values at a controller gain of $25 \% / \mathrm{Hz}$. As seen in the figures, increasing values of controller delay can cause significant frequency excursions.

Figure 12 shows plots of frequency at a location near Grand Coulee for the uncontrolled case $(\mathrm{Kp}=0$, $\mathrm{Td}=0)$ and for one of the cases shown in the two previous figures $(\mathrm{Kp}=25 \%, \mathrm{Td}=0.5)$. As seen in the figure, M1 (frequency nadir) shows improvement for the $25 \%$ gain case even while the delay effect of the controller begins to reduce the damping of the oscillatory mode. It is reasonable to conclude from Figure 12 that autonomous demand response incorporating significant delay characteristics might promote oscillatory instability even as it simultaneously improves frequency response. 


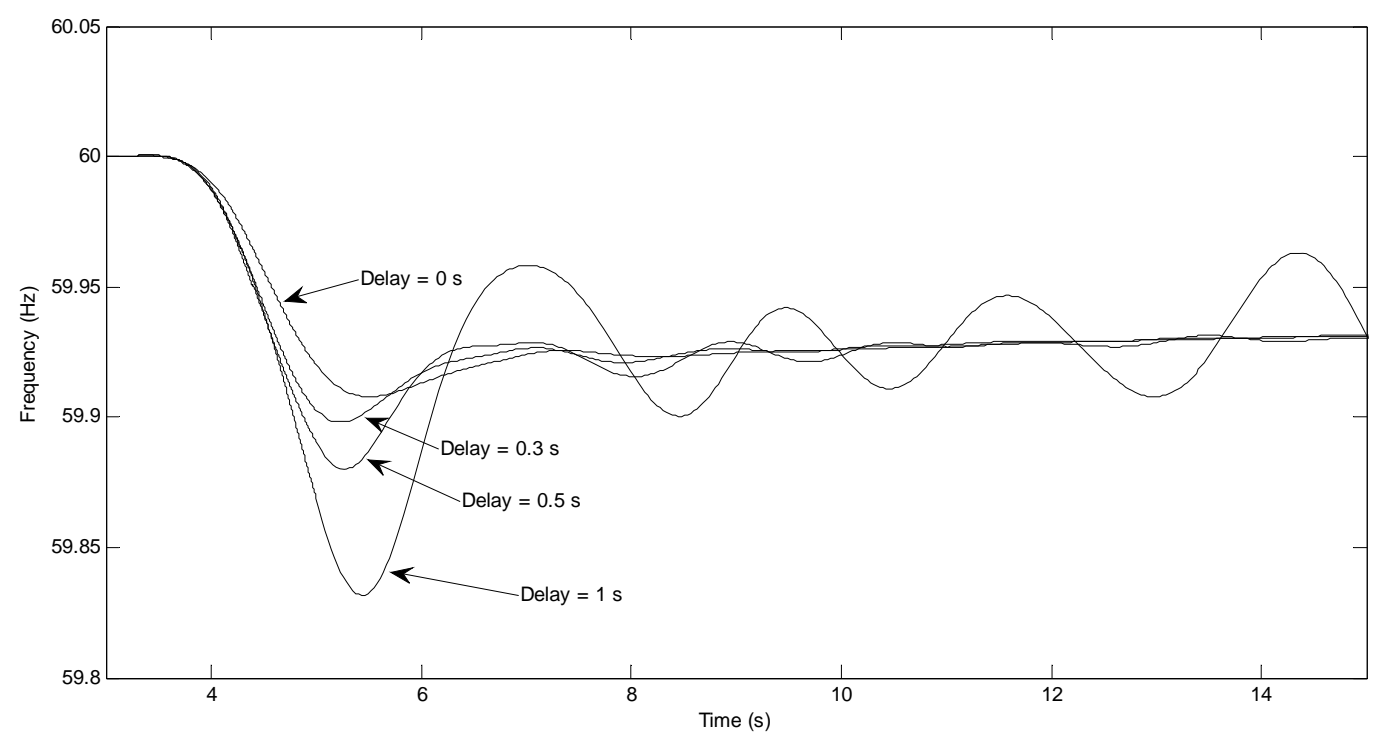

Figure 11. Various Delays Showing Instability

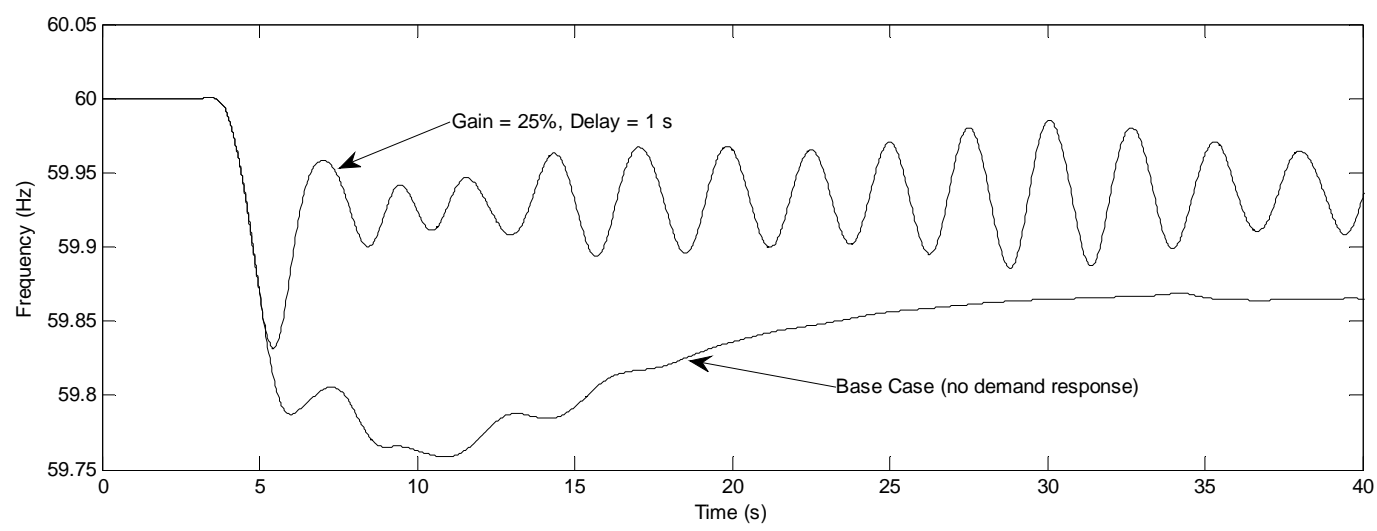

Figure 12. Comparison of Baseline to Demand Response with $\mathrm{Kp}=25 \%$, Delay $=1 \mathrm{~s}$

\subsection{Sensitivity to Block Size}

Block size relates to the "granularity" of the control action. Consider an example in which a utility wishes to implement autonomous load control at the feeder level in a fashion analogous to modern day under-frequency load shedding. In this example, the utility might choose feeders with an average load of $5 \mathrm{MW}$ and might trip these feeders based on the control law outlined earlier in this report. The block size parameter in the control law would be set to $5 \mathrm{MW}$, and load would respond to measured frequency changes in blocks of $5 \mathrm{MW}$ per step rather than in a continuous fashion. This example is somewhat contrived because of the chosen feeder level. The salient issue is whether or not shedding such large blocks of load using the feedback control law previously described might prove harmful to system performance as measured by the chosen metrics.

Figure 13 shows a set of frequencies near Grand Coulee for several block sizes ranging from 0 to 10 MW at a gain of $25 \%$. The baseline condition, absent an autonomous demand response, is also depicted 
in the figure for reference purposes. A block size of $0 \mathrm{MW}$ implies that an infinitesimally small load can respond to an infinitesimally small frequency deviation.

Two observations might help the reader interpret the time series data depicted in Figure 13. First, as frequency begins to decline in response to the loss of generation, it takes some time for the controller to aggregate enough load to implement the shedding of a full block. This time lag allows frequency to further decline before load response has the opportunity to counteract the generation/load imbalance. Second, the lower frequency nadir for larger block sizes forces a larger governor response and therefore the settling frequency is lower for large block sizes than for small. From the figure, the following qualitative conclusions can be drawn: 1) block size, or quantization, does not present a significant stability problem; 2) implementing autonomous demand response on large blocks of load does not provide as great a benefit for frequency response as implementing the strategy on smaller blocks of load; and 3) even with large block sizes, a substantial improvement in frequency response can be realized.

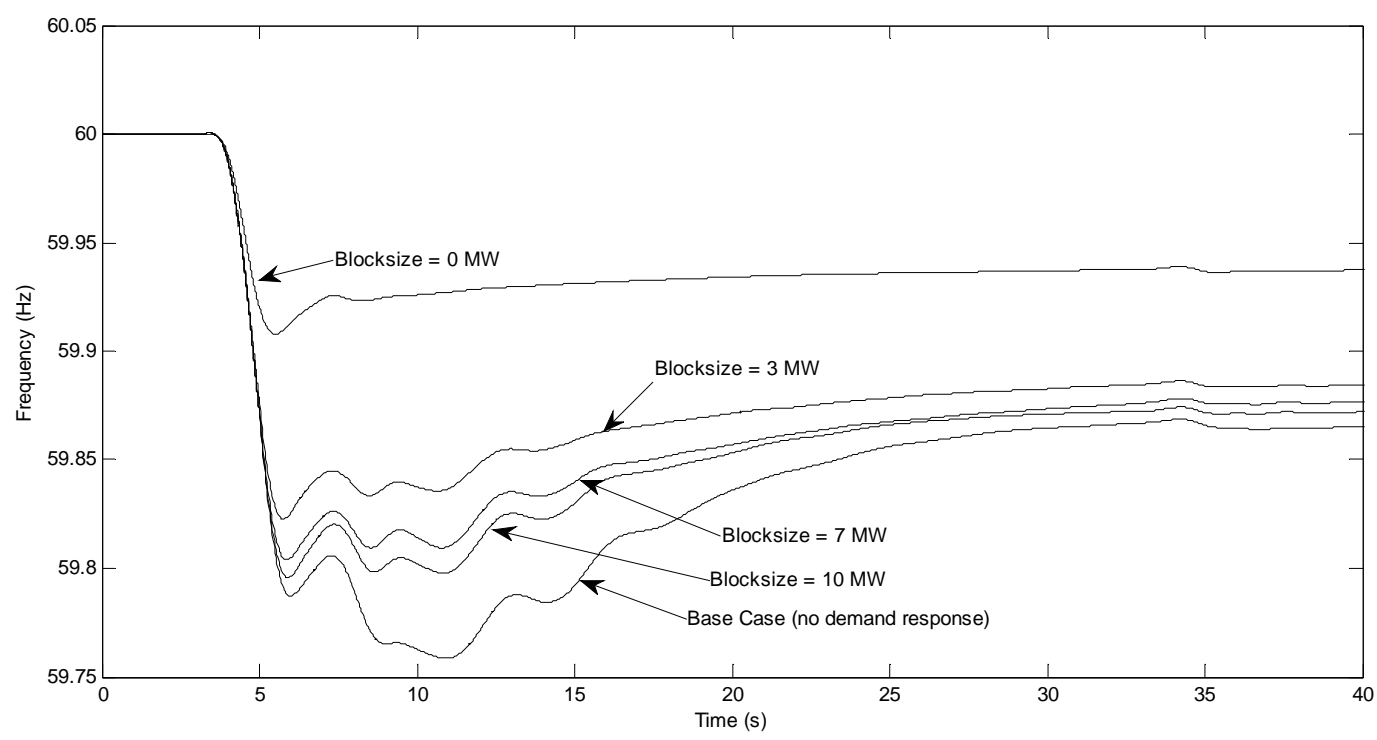

Figure 13. Various Sizes of Demand Response Blocks for $\mathrm{Kp}=\mathbf{2 5 \%}$

Figure 14 shows the sensitivities to metrics M1 through M5 for changes in block size. Metrics M1 (frequency nadir), M2 (settling frequency), M3 (time of settling) and M4 (nadir-based frequency response) are shown to be highly sensitive to changes in block size through a block size value of approximately $2 \mathrm{MW}$. For block sizes greater than $2 \mathrm{MW}$, the sensitivities to the relevant metrics remain relatively constant at a value of nearly zero. Block size does not appear to impact M5 (COI flow). The research team attributed the anomalies between block sizes of $2.5 \mathrm{MW}$ and $4 \mathrm{MW}$ to system nonlinearities; however, the exact cause of these anomalies remains unclear. 

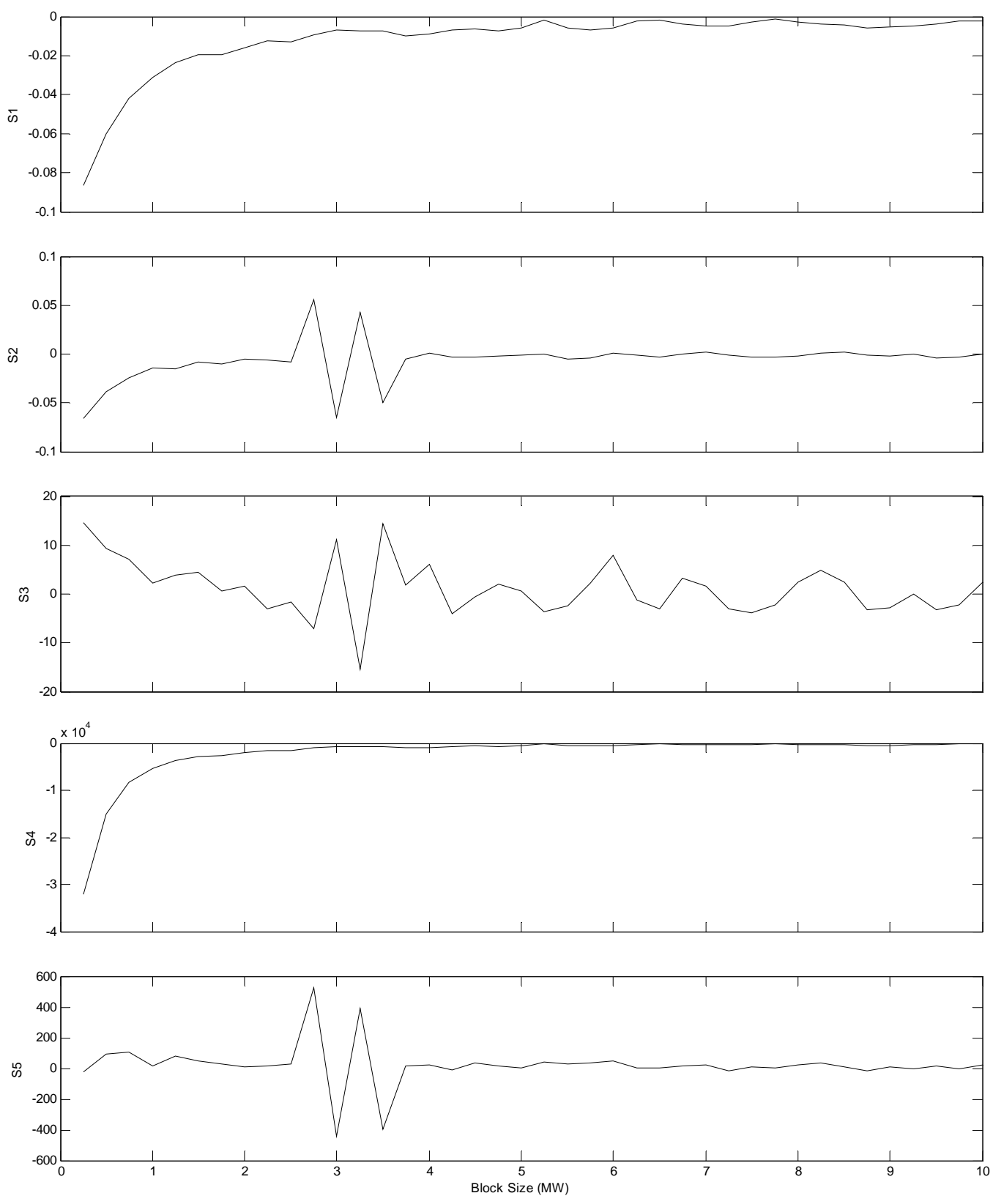

Figure 14. Sensitivities to Block Size

\subsection{Sensitivity to Localization}

Location plays a key role in frequency response. Prior to performing the studies, the study team postulated that autonomous demand response located close to the lost generation would provide the greatest benefit for frequency response. It was believed that because responsive loads closest to lost generation would "see" a decline in frequency more quickly than distant loads, this faster response time would lead to improvements in M1 (frequency nadir). We particularly believed that metric M5 (tie-line deviation from schedule) and the time of the frequency nadir (not a proposed metric) would be greatly 
impacted. M5 should be impacted because the load in the imbalanced area would be the first to "see" and, therefore the first to respond to the frequency excursion, thereby positively impacting the balancing authority's ability to maintain schedules. These expected effects were not entirely supported by the study results.

Figure 15 shows frequency at Grand Coulee for two different simulations, one in which autonomous demand response was implemented only in Arizona and the other for which autonomous demand response was implemented only in Alberta. In both cases, the contingency is a loss of two generating units in Arizona totaling $2800 \mathrm{MW}$. For the Arizona case, gain for responsive loads in Arizona was set to $60 \% / \mathrm{Hz}$, while for the Alberta case, gain was set at $90 \% / \mathrm{Hz}$. These gain settings resulted in total MW demand response being approximately equal for both cases.

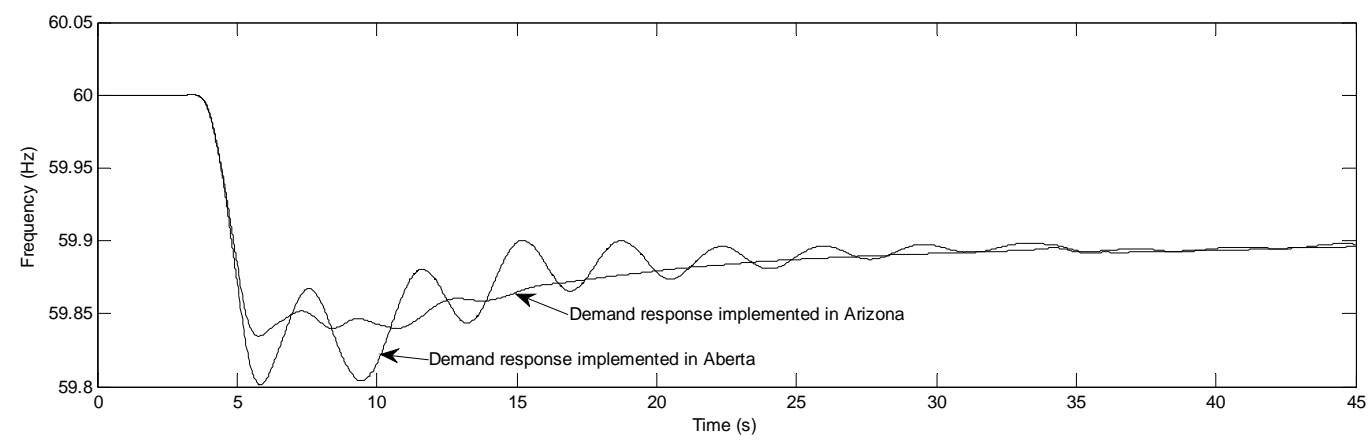

Figure 15. Effect of Localization on Frequency Response

Two salient conclusions can be drawn through examination of the simulation results shown in Figure 15. First, it is clear that localization of an autonomous demand response program may lead to reduced damping ratios for one or more oscillatory modes within the WECC. Second, localization does not appear to impact frequency response when one considers that M1 for this study uses a filtered frequency signal that "washes out" oscillatory instability. In fact, M1 for the localization-in-Arizona case was determined to be $59.8441 \mathrm{~Hz}$, while M1 for the localization-in-Alberta case was $59.8382 \mathrm{~Hz}$, a difference of only $6 \mathrm{mHz}$. In summary, it proved to be true that responsive loads in close proximity to a loss of generation responded more quickly than responsive loads at a distance from the affected generator. This time lag effect had a detrimental impact on stability in the same manner as that of a "pure" time delay (as discussed in Section 6.2); however, the postulation that the time lag effect would also have a detrimental impact on frequency response did not prove to be true.

Figure 16 shows COI flow for the two simulations described above using the same contingency, namely a loss of two Palo Verde generating units. With M5 defined as the change in COI flow normalized to the magnitude of the lost generation, a marked improvement in M5 as a result of localization of autonomous demand response is readily apparent in the figure. As postulated, localization has a significant impact on M5. 


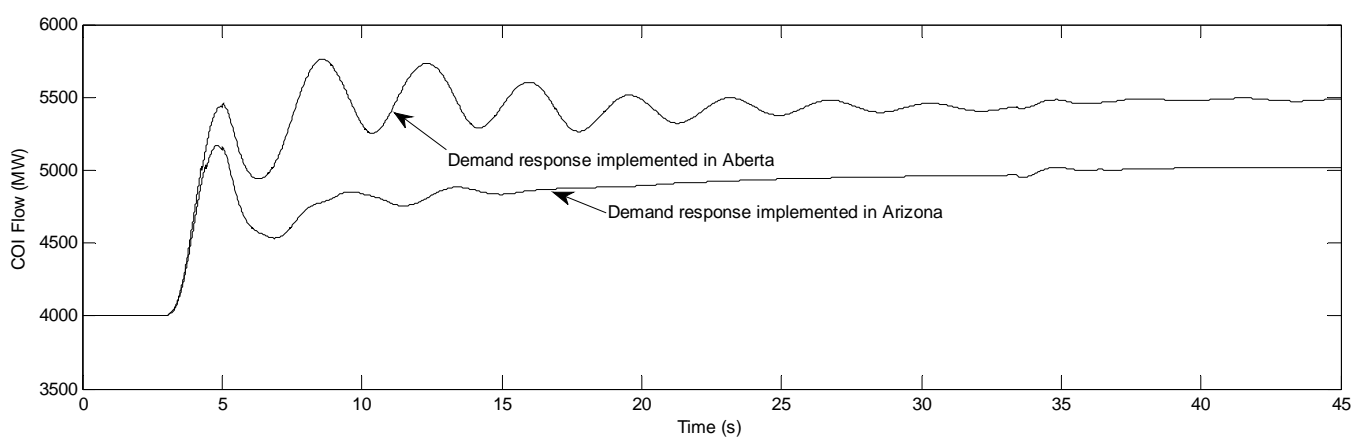

Figure 16. COI Flow for Arizona Implementation versus Alberta Implementation

\subsection{Sensitivity to Inhibit of Restoration}

The term "Inhibit of Restoration" refers to a characteristic of many end-use appliances. Once turned off, some of these devices will not start to operate until some time has gone by, even if power is applied almost immediately. In all previous examples, the load control algorithm allowed the load to respond in equal proportion to either an increase or a decrease in system frequency. This portion of the project sought to quantify the effect of controllers that can rapidly shed load from the system but cannot rapidly restore load to pre-disturbance conditions.

Figure 17 shows the total load response to a loss of two Palo Verde units in the 2009HW base case for simulations with the inhibit function enabled and disabled, both at a gain of $30 \% / \mathrm{Hz}$. Frequency at Grand Coulee for the same contingency is shown below the total load response for comparison.

In addition to demonstrating the effect of enabling the inhibit of restoration flag within the control law, the figure shows that significant governor action is taking place in tandem with the autonomous demand response. Note that after approximately $t=6 \mathrm{~s}$, the demand response has reached its maximum for both the "inhibit enabled" and "inhibit disabled" simulations. For the "inhibit enabled" case, load no longer participates in the frequency response after the 6 second mark, yet the system continues to accelerate, as evidenced by the advancing frequency, through the 30 second mark. This phenomenon is attributed to governor response that continues to add more real power to the system even after the supplydemand imbalance is initially restored at the frequency nadir. 

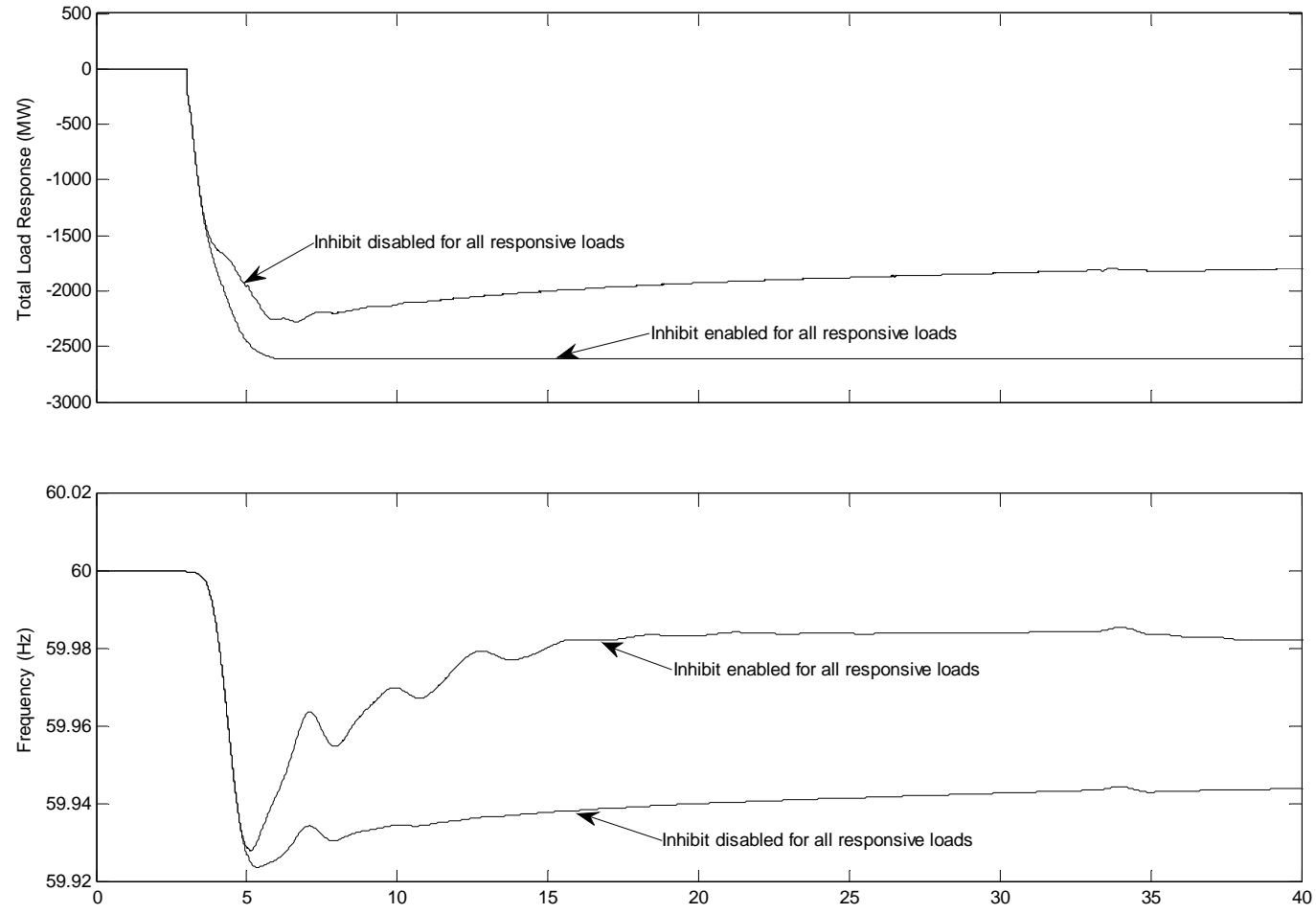

Figure 17. Control Action for Inhibit Function Using 2009HW Base Case

Figure 18 shows what might be considered a detrimental effect of governor response continuing after a significant amount of demand response has been deployed. The figure shows three traces of frequency at a location near Grand Coulee Dam for a loss of two Palo Verde units on the 2009HW base case. The effect of governor response following full deployment of "inhibit enabled" load can be readily observed. In the extreme, the simulations show that for extremely high penetration of autonomous demand response in the absence for coordination with conventional generation, there is a possibility that frequency might overshoot the intended target of $60 \mathrm{~Hz}$.

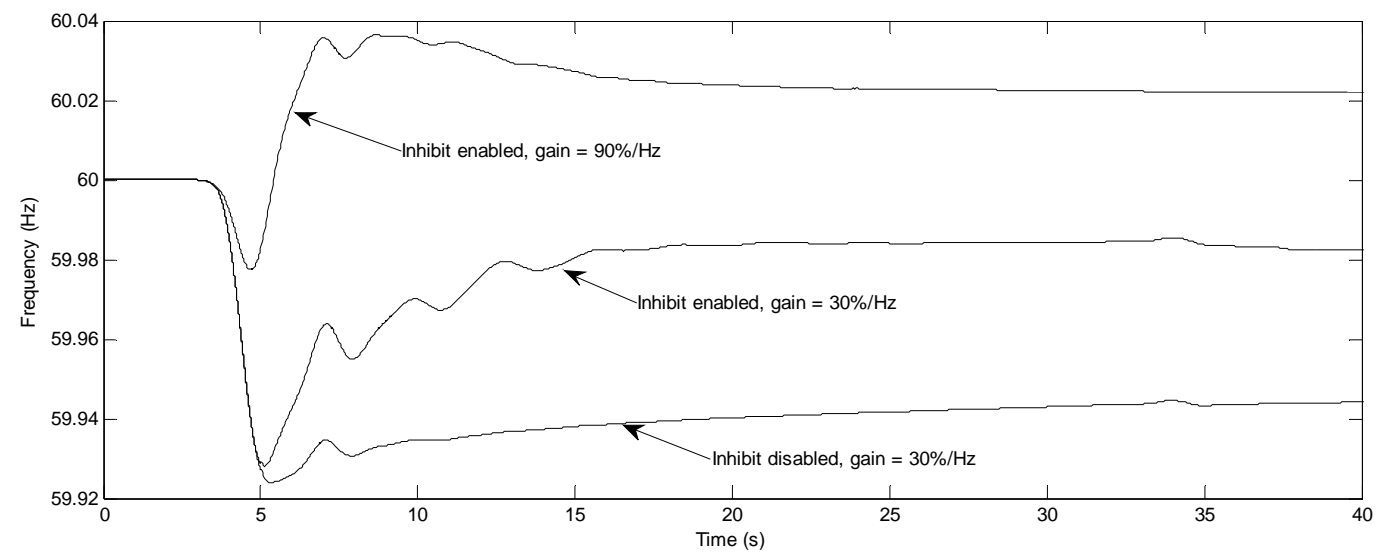

Figure 18. Effect of Inhibit of Restoration Function Showing Frequency Overshoot 
The physical significance of overshoot, in which M2 (settling frequency) exceeds $60 \mathrm{~Hz}$, is that the combined response of both autonomous demand response plus classical primary frequency control (i.e., generator governor action) exceeds the megawatt value of the lost generation in the original contingency. When the Inhibit function is active, loads that have been shed cannot rapidly respond as frequency recovers. Therefore the burden of restoring system frequency to nominally $60 \mathrm{~Hz}$ falls to the generators. For extremely high levels of autonomous demand response the magnitude of the load response as frequency declines is roughly equal to the magnitude of the lost generation. In this case the primary frequency response provided by classical generation causes a sustained over-frequency condition.

As stated above, the differentiating factor observed by enabling the inhibit function is the overshoot seen in M2, the settling frequency. No remarkable differences are observed in the other four metrics when compared to the cases where the inhibit function is disabled. Figure 19 shows M2 (settling frequency) as a function of gain with the inhibit function turned on for a loss of two Palo Verde generators on the $2009 \mathrm{HW}$ base case. Note that, for this contingency, the system does not overshoot 60 $\mathrm{Hz}$ until autonomous demand response exceeds penetration levels corresponding to $50 \% / \mathrm{Hz}$.

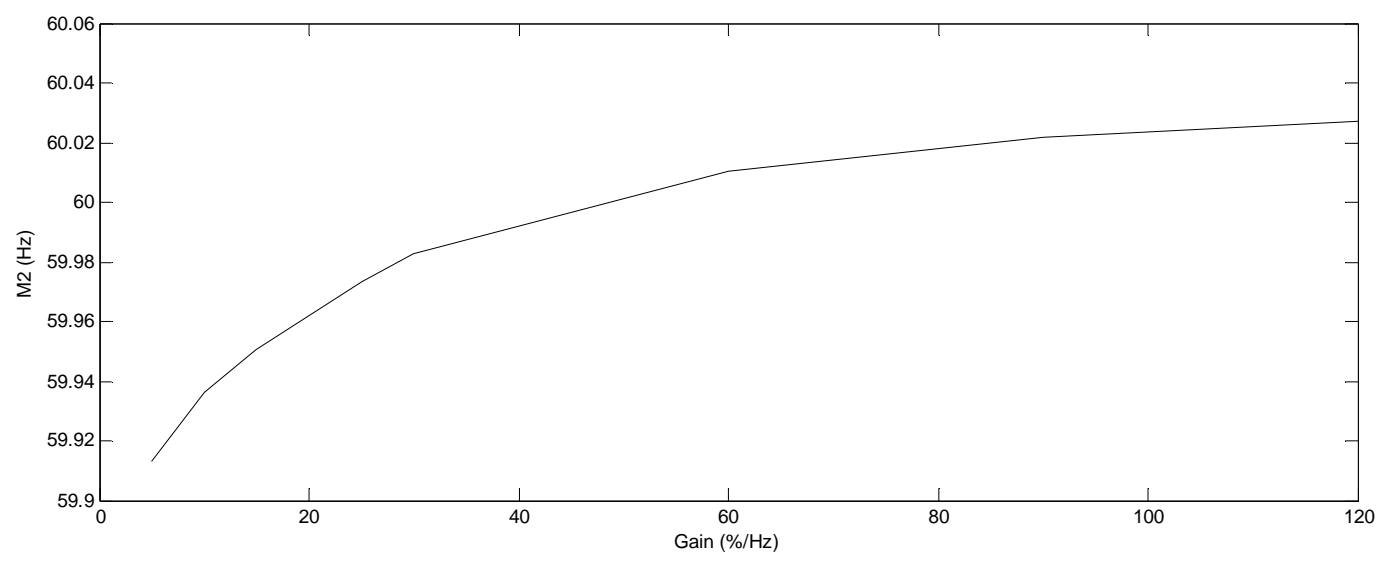

Figure 19. M2 for Inhibit Sensitivity 



\subsection{Conclusions}

This research sought to achieve two objectives: to apply a simple autonomous demand response control law to a large, realistic base case where it had previously only been applied to reduced-order models; and to attempt to quantify the benefit of responsive load to the problem of achieving an adequate frequency response for a large interconnected system. The study team also wished that an additional outcome of the research would be generic models for an autonomous demand response controller implemented in a commercial transient stability simulation tool for use in future research on this subject.

The study generated a large amount of data. More than 6000 simulations were conducted. Results from each simulation were tabulated and categorized into one of five study categories, each representing a different parameter within the control law. In this manner it was possible to examine the effect of changing a single controller parameter and attempting to quantify the impact of this change on the frequency response of the interconnected grid.

Five metrics were developed to help assess the benefit to frequency response of an interconnected power system. Application of these metrics to each of the five study categories was not straightforward, and adjustments and alternative observations were made as needed in these cases.

We briefly discuss our significant conclusions in the following sections.

\subsection{General Conclusions}

As postulated in previous work [5-8], this study demonstrates that autonomous demand response can provide substantial benefit by responding to under-frequency events in the interconnected power system. Further, the study demonstrates that the characteristics of frequency response delivered by autonomous demand response are analogous to generator governor action.

\subsection{Gain Sensitivities}

Generally, the study shows improvements in all five metrics as controller gain is increased. Specifically, we draw the following conclusions:

- No differences were found between controller gain and level of participation for autonomous demand response. Increasing the gain on a controller acting on an aggregated load model is equivalent to increasing the participation, or penetration, level of a properly coordinated autonomous demand response program. In practice, increased gain levels will be achieved by greater participation levels from sponsored appliances or end uses.

- It proved impossible to create instabilities or to degrade frequency response only by increasing controller gain. Using combinations of gain and delay or block size, we were able to create problems that are discussed in more detail below. If delays are small and known and block size is small, as would be the case for any practical implementation, the penetration levels of autonomous demand response controllers should not have a detrimental impact on reliability.

- There appeared to be diminishing returns for gains above 25 to 30 percent. The sensitivities of each of the metrics peaked in this region of gain settings. In practical terms, this means that putting 
anything in excess of 25 percent of total system load under an autonomous demand response scheme may not be worthwhile. It is unlikely that this point would ever be reached, and our studies show that as little as 3 percent of total system load under control yields substantial improvements in frequency response.

\subsection{Delay Sensitivities}

Controller delay proved to be the most serious issue associated with widespread deployment of autonomous demand response controllers. Delay can be introduced by instrumentation and sensing equipment and/or by actuators. An actuator associated with a common household appliance would typically be a contactor within the controlled appliance.

For a fixed gain, any amount of delay reduced the effectiveness of the demand response scheme below what was observed for the same gain with no delay, as measured in each of the five metrics. Even with the introduction of a significant amount of delay, the metrics related to frequency response continued to show improved frequency response when compared to the "no control" case; however, large delays did seriously impact system reliability by degrading the damping of the oscillatory modes of the system. Minimal delays, on the order of $0.1 \mathrm{~s}$, were considered to be acceptable, and anything less than approximately $0.25 \mathrm{~s}$ proved to be "safe" with respect to oscillatory instability.

We further conclude that delays exceeding $0.5 \mathrm{~s}$ can result in system instability when tested on the western power grid. Therefore, to minimize delays, it would be extremely important to work with manufacturers who intend to incorporate autonomous demand response into their appliances.

\subsection{Block Size Sensitivities}

Increasing the block size did not significantly change the performance of the overall scheme, as evidenced by the five assessment metrics. Block size, whether very small or very large, did not appear to introduce a significant stability problem.

\subsection{Locational Sensitivities}

Assessing the impact of location on frequency response is important in that autonomous demand response programs may initially be implemented locally rather than evenly across the entire system. This would be the case if, for example, one utility were an early adopter of the technology and this utility experienced high levels of participation in the program before other regional utilities implemented similar programs. The study results demonstrate that location has relatively little impact on frequency response, but that stability problems similar to those encountered within the delay studies may be observed for high penetration levels in one location.

In terms of frequency response, the five assessment metrics showed very little difference between 100 MW of response in one location versus a similar magnitude response in another location. This can be explained in that the frequency nadir of filtered frequency signals at various locations in the system remains relatively constant; therefore, loads in each region of the grid can expect to see generally the same magnitude of change in frequency at that location. 
The timing of the frequency response varies from one location to another, and this observation was one of the expected outcomes of the study. The timing of the frequency response was the key factor causing oscillatory instability in some cases. When autonomous demand response is deployed in one region and a system event subsequently causes a frequency deviation at a distant location, oscillatory damping is adversely affected. The effect is due to the inherent time lag between the time that a supplydemand imbalance first occurs and the time at which the responsive loads "see" the frequency excursion.

Finally, the study results show that M5 (change in COI flow) was significantly impacted by the location of the demand response. Because loads in close proximity to lost generation respond more rapidly than loads remote from the lost generation, the balancing authority in which an autonomous demand response program has been deployed and a loss of generation subsequently occurs would enjoy a benefit in terms of maintaining scheduled flows. Deviations from scheduled tie-line flows are minimized and overall frequency response improves.

\subsection{Inhibit Load Restoration Sensitivities}

When loads cannot be restored to their pre-disturbance values after having been shed by an autonomous demand response controller, the primary effect is an "improvement" in metric M2 (settling frequency). "Improvement" is enclosed in quotation marks because there can be extreme cases in which M2 settles at a value greater than $60 \mathrm{~Hz}$, in which case we chose to call these "overshoot" cases. Overshoot can happen at extreme gain values when all responsive loads cannot be restored to their predisturbance value. Overshoot was not observed at gain values less than approximately $50 \% / \mathrm{Hz}$. Therefore, the appropriate conclusion is that the inhibit function would not cause overshoot in any practical situation.

The study also shows that inhibiting the restoration of load in response to an increase, following a decline, in measured frequency beneficially impacted oscillatory stability problems observed when operating with long delay times. The responsive load, with inhibit enabled, does not participate in oscillatory dynamics but rather simply "sheds" in the same fashion that would be observed in a traditional under-frequency load shedding scheme. 


\subsection{Further Research}

Because of the substantial volume of information generated during this project, we identified the following areas that warrant further research:

- Determine the voltage effects of autonomous demand response, particularly when the controller manipulates both real and reactive portions of load.

- Determine how classical governors might be modified to accommodate increasing penetration levels of autonomous demand response controllers.

- Determine if there is a point where the supply side need not be burdened with providing frequency responsive reserve.

- Develop possible implementation strategies and determine how benefits can be accrued to participants.

- Given a specific set of controller delay parameters, determine if there is an optimal penetration level within which the incremental benefit of adding one additional megawatt of demand response provides a declining benefit to the sponsoring entity. We saw some indications that there is an optimal gain level, in the sense that additional amounts of control yielded declining benefit.

- Develop a way to better observe the effects of localization. Redesigning the controller so it provides an adaptive gain that varies in proportion to the overall amount of load present in the area or zone might be an implementable approach. 


\subsection{References}

[1] North American Electric Reliability Corporation. 2011. Glossary of Terms Used in Reliability Standards. North American Electric Reliability Council, Atlanta, Georgia. Available http:/www.nerc.com/files/Glossary_of_Terms_2011Mar15.pdf. Updated March 15, 2011. Accessed (June 2011)

[2] Federal Energy Regulatory Commission. 2009. Possible Elements of a National Action Plan on Demand Response - A Discussion Draft. October. Docket No. AD09-10, Federal Energy Regulatory Commission Staff, Federal Energy Regulatory Commission, Washington, D.C.

[3] Western Electricity Coordinating Council. 2005. White Paper: Frequency Responsive Reserve Standard. April 7. Western Electricity Coordinating Council Reserve Issues Task Force, Salt Lake City, Utah.

[4] Eto J, J Undrill, P McMakin, R. Daschmans, B Williams, B Haney, R Hunt, J Ellis, H Illian, C Martinez, M O'Malley, K Coughlin, and KH Lacommare. December 2010. Use of Frequency Response Metrics to Assess the Planning and Operating Requirements for Reliable Integration of Variable Renewable Generation. LBNL-4142E, Lawrence Berkeley National Laboratory, Berkeley, California. Available at http://certs.lbl.gov/pdf/lbnl-4142e.pdf.

[5] Trudnowski, D, M Donnelly, and E Lightner. May 2006. "Power-System Frequency and Stability Control using Decentralized Intelligent Loads," in Proceedings of the 2005 IEEE T\&D Conference and Expo, Dallas, Texas, IEEE, Piscataway, New Jersey.

[6] B. Kirby. March 2003., "Spinning Reserve From Responsive Loads," ORNL/TM-2003/19, Oak Ridge National Laboratory, Oak Ridge, Tennessee.

[7] Donnelly M, D Harvey, R Munson, and D Trudnowski. 2010. "Frequency and Stability Control using Decentralized Intelligent Loads: Benefits and Pitfalls." In Proceedings. of the 2010 PES General Meeting, Minneapolis, Minnesota.

[8] Makarov YV, J Ma, S Lu, and TB Nguyen. 2008. Assessing the Value of Regulation Resources Based on Their Time Response Characteristics." PNNL-17632, Pacific Northwest National Laboratory, Richland, Washington.

[9] GE Energy. 2008. PSLF User's Manual Version 17. September 8. GE Energy, Energy Consulting Software. Website:

http://www.ge-energy.com/products_and_services/products/energy_consulting_software/.

[10] PJM Interconnection. 2010. Load Management Performance Report 2009/2010. PJM, Norristown, Pennsylvania. Available at http://www.pjm.com/documents.aspx. Accessed June 2011. 
[11] U.S. Department of Energy. 2006. Benefits of Demand Response in Electricity Markets and Recommendations for Achieving Them, A Report to the United States Congress Pursuant to Section 1252 of the Energy Policy Act of 2005. February 2006. U.S. Department of Energy, Washington, D.C. Available at http://www.eetd.lbl.gov/ea/ems/reports/congress-1252d.pdf.

[12] WECC. 2006. 10-Year Coordinated Plan Summary (2006-2015). July 2006. Western Electricity Coordinating Council, Salt Lake City, Utah. Available at http://www.wecc.biz/library/WECC Documents/Publications/10-Year Coordinated Plan Summaries/2006-2015 10-Year Coordinated Plan Summary.pdf.

[13] Illian H. December 2010. Frequency Control Performance Measurement and Requirements. LBNL-4145E, Lawrence Berkeley National Laboratory, Berkeley, California,.

[14] Federal Energy Regulatory Commission. 2011. Use of Frequency Response Metrics to Assess the Planning and Operating Requirements for Reliable Integration of Variable Renewable Generation. slides presented to the NERC Technical Issues Subcommittee, Charlotte, NC, February 9-10, 2011.

[15] Slides for FR Technical Conference. [Online]. Available http://www.ferc.gov/EventCalendar/Files/20100923090101-FERC Staff Slides.ppt. Accessed June 2011.

[16] Lemmons D. 2008. “WECC Frequency Responsive Reserve Criterion Proposal.” Slides presented at WECC FRR Educational Workshop, November 14, 2008, Portland, Oregon. Available at http://www.wecc.biz/Standards/Development/wecc0044/Lists/Calendar/DispForm.aspx?ID=12.

[17] Armke E. "BAL-001-TRE-1 Primary Frequency Response Evaluation Model Version 1.9 Manual," [Online]. Available: http://texasre.org/Standards Tracking Documents/Evaluation Model Manual Final Clean 20100831.pdf . Accessed June 2011.

[18] "Frequency Response Characteristic Survey Training Document," in NERC Operating Manual, North American Electric Reliability Corporation, Washington, D.C., March 2008.

[19] Frequency Response Standard Whitepaper. Prepared by the Frequency Task Force of the NERC Resources Subcommittee, Princeton, NJ. April 6, 2004.

[20] NERC Resources Subcommittee, "Balancing and Frequency Control," NERC training document, Princeton, NJ, July 5, 2009.

[21] Schweppe Fred C., "Frequency adaptive, power-energy re-scheduler," U.S. Patent 4,317,049, February 23, 1982.

[22] Chassin et al. GridLAB-D Source Code and Documentation, Pacific Northwest National Laboratory. Available at http://www.gridlabd.org/. 
Appendix A

\section{Selected Data for Gain Sensitivities}





\section{Appendix A - Selected Data for Gain Sensitivities}

This appendix describes selected results from the study. The results shown are from simulations on the 2009HSP base case in which two Palo Verde generating units were taken out of service as a representative contingency. Gain is expressed in units of $\% / \mathrm{Hz}$, so a gain of 30 in a system with TtlMWLoads $=106,000 \mathrm{MW}$ would correspond to a gain of $3180 \mathrm{MW} / 0.1 \mathrm{~Hz}$.

Table A.1. Gain Metrics for 2PV on 2009HSP Case

\begin{tabular}{|c|c|c|c|c|c|c|c|c|c|c|c|}
\hline Gain & M1 & M2 & M3 & M4 & M5 & S1 & S2 & S3 & S4 & S5 & $\begin{array}{c}\text { Total MW } \\
\text { Controlled }\end{array}$ \\
\hline 0 & 59.77 & 59.87 & 28.90 & 12009 & 1273.7 & 0.0000 & 0.0000 & 0.0000 & 0.0 & 0.0 & 0.0 \\
\hline 3 & 59.81 & 59.88 & 29.13 & 14792 & 1194.6 & 0.0146 & 0.0046 & 0.0778 & 927.6 & -26.4 & -618.5 \\
\hline 6 & 59.84 & 59.89 & 27.90 & 17696 & 1133.1 & 0.0104 & 0.0040 & -0.4111 & 968.2 & -20.5 & -1038.0 \\
\hline 9 & 59.86 & 59.90 & 26.23 & 20567 & 1082.9 & 0.0074 & 0.0033 & -0.5556 & 956.8 & -16.7 & -1369.5 \\
\hline 12 & 59.88 & 59.91 & 25.57 & 23390 & 1045.4 & 0.0055 & 0.0030 & -0.2222 & 1.2 & -12.5 & 623.2 \\
\hline 15 & 59.89 & 59.92 & 23.73 & 26220 & 1011.5 & 0.0043 & 0.0023 & -0.6111 & 943.3 & -11.3 & -1819.6 \\
\hline 18 & 59.90 & 59.93 & 22.00 & 29071 & 980.6 & 0.0035 & 0.0020 & -0.5778 & 950.2 & -10.3 & -1973.4 \\
\hline 21 & 59.91 & 59.93 & 20.13 & 32448 & 970.1 & 0.0033 & 0.0021 & -0.6222 & 1125.6 & -3.5 & -2061.2 \\
\hline 24 & 59.92 & 59.94 & 18.60 & 35396 & 945.9 & 0.0024 & 0.0015 & -0.5111 & 982.7 & -8.1 & -2157.8 \\
\hline 27 & 59.93 & 59.94 & 17.10 & 38384 & 924.7 & 0.0021 & 0.0013 & -0.5000 & 996.0 & -7.0 & -2235.3 \\
\hline 30 & 59.93 & 59.94 & 15.63 & 41388 & 906.4 & 0.0018 & 0.0012 & -0.4889 & 1001.4 & -6.1 & 311.0 \\
\hline 33 & 59.94 & 59.95 & 14.27 & 44399 & 891.4 & 0.0015 & 0.0010 & 556 & 1003.9 & -5.0 & 376.1 \\
\hline 36 & 59.94 & 59.95 & 13.07 & 47429 & 879.3 & 0.0013 & 0.0009 & -0.4000 & 1009.8 & -4.0 & -2430.2 \\
\hline 39 & 59.95 & 59.95 & 13.07 & 50477 & 872.0 & 0.0012 & 0.0008 & 0.0000 & 1016.0 & -2.4 & -2476.6 \\
\hline 42 & 59.95 & 59.95 & 13.07 & 53549 & 865.3 & 0.0011 & 0.0008 & 0.0000 & 1023.9 & -2.3 & -2515.8 \\
\hline 45 & 59.95 & 59.96 & 13.07 & 56617 & 859.3 & 0.0009 & 0.0007 & 0.0000 & 1022.9 & -2.0 & -2550.6 \\
\hline 48 & 59.95 & 59.96 & 13.07 & 59703 & 854.0 & 0.0009 & 0.0006 & 0.0000 & 1028.5 & -1.8 & -2580.7 \\
\hline 51 & 59.96 & 59.96 & 13.07 & 62793 & 849.5 & 0.0008 & 0.0006 & 0000 & 1030.1 & -1.5 & -2607.0 \\
\hline 54 & 59.96 & 59.96 & 13.07 & 65895 & 845.1 & 0.0007 & 0.0005 & 0.0000 & 1033.9 & -1.5 & -2629.8 \\
\hline 57 & 59.96 & 59.96 & 13.07 & 69005 & 841.2 & 0.0006 & 0.0005 & 0.0000 & 1036.6 & -1.3 & -2649.9 \\
\hline 60 & 59.96 & 59.97 & 13.07 & 72117 & 837.5 & 0.0006 & 0.0005 & 0.0000 & 1037.3 & -1.2 & -2667.7 \\
\hline 63 & 59.96 & 59.97 & 13.07 & 75241 & 834.2 & 0.0005 & 0.0004 & 0.0000 & 1041.3 & -1.1 & -2683.3 \\
\hline 66 & 59.96 & 59.97 & 13.07 & 78372 & 831.0 & 0.0005 & 0.0004 & 0.0000 & 1043.9 & -1.1 & -2697.3 \\
\hline 69 & 59.97 & 59.97 & 13.07 & 81511 & 828.1 & 0.0005 & 0.0004 & 0.0000 & 1046.5 & -0.9 & -2709.7 \\
\hline 72 & 59.97 & 59.97 & 13.07 & 84636 & 825.4 & 0.0004 & 0.0003 & 0.0000 & 1041.4 & -0.9 & -2721.4 \\
\hline 75 & 59.97 & 59.97 & 13.07 & 87769 & 822.9 & 0.0004 & 0.0003 & 0.0000 & 1044.4 & -0.8 & -2732.1 \\
\hline 78 & 59.97 & 59.97 & 13.07 & 90909 & 820.6 & 0.0004 & 0.0003 & 0.0000 & 1046.8 & -0.8 & -2741.5 \\
\hline 81 & 59.97 & 59.97 & 13.07 & 94058 & 818.4 & 0.0003 & 0.0003 & 0.0000 & 1049.5 & -0.7 & -2750.3 \\
\hline 84 & 59.97 & 59.97 & 13.07 & 97212 & 816.3 & 0.0003 & 0.0003 & 0.0000 & 1051.5 & -0.7 & -2758.1 \\
\hline 87 & 59.97 & 59.97 & 13.07 & 100370 & 814.2 & 0.0003 & 0.0003 & 0.0000 & 1051.2 & -0.7 & -2765.9 \\
\hline 90 & 59.97 & 59.97 & 13.07 & 103520 & 812.1 & 0.0003 & 0.0002 & 0.0000 & 1050.1 & -0.7 & -2772.8 \\
\hline 93 & 59.97 & 59.98 & 13.07 & 106670 & 810.2 & 0.0003 & 0.0002 & 0.0000 & 1053.0 & -0.6 & -2779.1 \\
\hline 96 & 59.98 & 59.98 & 13.07 & 109820 & 808.4 & 0.0003 & 0.0002 & 0.0000 & 1048.8 & -0.6 & -2785.5 \\
\hline 99 & 59.98 & 59.98 & 13.07 & 112990 & 806.7 & 0.0002 & 0.0002 & 0.0000 & 1054.7 & -0.6 & -2806.4 \\
\hline 102 & 59.98 & 59.98 & 13.07 & 116150 & 805.2 & 0.0002 & 0.0002 & 0.0000 & 1054.5 & -0.5 & -2836.1 \\
\hline
\end{tabular}


Table A.2. Delay Metrics for 2PV on 2009HSP Case

\begin{tabular}{|c|c|c|c|c|c|c|c|c|c|c|c|c|}
\hline Gain & $\begin{array}{l}\text { Delay } \\
\text { (s) }\end{array}$ & M1 & M2 & M3 & M4 & M5 & S1 & S2 & S3 & S4 & S5 & $\begin{array}{l}\text { Total MW } \\
\text { Controlled }\end{array}$ \\
\hline 25 & 0 & 59.92 & 59.94 & 18.07 & 36390 & 937.7 & 0.0000 & 0.0000 & 0.000 & 0 & 0 & -2185.4 \\
\hline 25 & 0.1 & 59.92 & 59.94 & 18.00 & 36325 & 937.6 & -0.0014 & 0.0003 & -0.667 & -642 & -1 & -2207.4 \\
\hline 25 & 0.2 & 59.92 & 59.94 & 17.87 & 36234 & 936.9 & -0.0019 & -0.0001 & -1.333 & -912 & -7 & -2251.9 \\
\hline 25 & 0.3 & 59.92 & 59.94 & \begin{tabular}{|l|}
17.77 \\
\end{tabular} & 36113 & 936.2 & -0.0026 & 0.0003 & -1.000 & -1210 & \begin{tabular}{|l|}
-7 \\
\end{tabular} & -2310.0 \\
\hline 25 & 0.4 & 59.92 & 59.94 & 17.87 & 35371 & 919.0 & -0.0163 & -0.0101 & 1.000 & -7427 & -172 & -2409.3 \\
\hline 25 & 0.5 & 59.92 & 59.94 & 17.73 & 35112 & 917.5 & -0.0058 & 0.0000 & -1.333 & -2582 & -15 & -2488.4 \\
\hline 25 & 0.6 & 59.92 & 59.94 & 17.63 & 34762 & 900.4 & -0.0080 & -0.0001 & -1.000 & -3505 & -172 & -2569.4 \\
\hline 25 & 0.7 & 59.92 & 59.94 & 17.37 & 34291 & 913.4 & -0.0111 & -0.0003 & -2.667 & -4704 & 130 & -2658.3 \\
\hline 25 & 0.8 & 59.92 & 59.94 & 16.93 & 33693 & 1004.1 & -0.0145 & -0.0010 & -4.333 & -5983 & 907 & -2747.1 \\
\hline 25 & 0.9 & 59.92 & 59.94 & 19.80 & 32989 & 1118.7 & -0.0178 & 0.0136 & 28.667 & -7046 & 1145 & -2830.1 \\
\hline 25 & 1 & 59.91 & 59.94 & 33.43 & 32182 & 1302.4 & -0.0213 & 0.0484 & 136.330 & -8061 & 1837 & -2928.0 \\
\hline 25 & 1.1 & 59.88 & 59.88 & 40.30 & 22610 & -36.5 & -0.3684 & -0.6502 & 68.667 & -95724 & -13389 & -4774.0 \\
\hline 25 & 1.2 & 59.87 & \begin{tabular}{l|l}
59.88 \\
\end{tabular} & 33.77 & 21891 & -1593.3 & -0.0407 & -0.0155 & -65.333 & -7189 & -15568 & 5212.4 \\
\hline 25 & 1.3 & 59.87 & 59.98 & 44.30 & 21954 & -4498.2 & 37 & 1.0 & 105. & 630 & -29049 & 5457.7 \\
\hline 25 & 1.4 & 59.90 & 59.94 & 18.67 & 28533 & 1698.9 & 0.2941 & -0.3904 & -256.330 & 65792 & 61971 & 3414.6 \\
\hline 25 & 1.5 & 59.90 & \begin{tabular}{l|l|}
59.92 \\
\end{tabular} & 27.87 & 27591 & 1152.6 & -0.0335 & -0.2126 & 92.000 & -9425 & -5463 & 3537.7 \\
\hline 25 & 1.6 & 60.01 & 60.01 & 43.37 & -305210 & -6796.4 & 1.1066 & 0.8607 & 155.000 & -3328000 & -79490 & 5762.1 \\
\hline 25 & 1.7 & 59.58 & 59.58 & 41.50 & 637 & -5328.0 & -4.3104 & -4.3060 & -18.667 & 3118500 & 14684 & 747.1 \\
\hline 25 & 1.8 & 60.08 & 60.08 & 44.20 & -34026 & 204.1 & 5.0415 & 5.0415 & 27.000 & -406640 & 55321 & -13042.0 \\
\hline 25 & 1.9 & 60.20 & 60.20 & 39.60 & -14153 & -2976.2 & 1.1556 & 1.1556 & -46.000 & 198740 & -31803 & -330.9 \\
\hline 25 & 2 & 59.85 & 59.93 & 14.47 & 19195 & 250.6 & -3.4371 & -2.7250 & -251.330 & 333480 & 32268 & -5690.4 \\
\hline 25 & 2.1 & 59.82 & 59.82 & 44.43 & 15796 & -1452.5 & -0.3139 & -1.0260 & 299.670 & -33993 & -17031 & 6420.4 \\
\hline 25 & 2.2 & 59.80 & 59.83 & 42.27 & 14251 & 37.4 & -0.1921 & 0.0440 & -21.667 & -15447 & 14899 & 9616.9 \\
\hline 25 & 2.3 & 59.87 & 59.93 & 15.67 & 21361 & 289.9 & 0.6540 & 0.98 & -266.000 & 71101 & 2525 & 4508.1 \\
\hline 25 & 2.4 & 59.87 & 59.97 & 17.50 & 0778 & 1512.0 & -0.0368 & 0.43 & 18.333 & -5833 & 12221 & -4617.1 \\
\hline 25 & 2.5 & 59.86 & 59.97 & 17.87 & 20235 & 1489.1 & -0.0361 & 0.0442 & 667 & -5424 & -22 & -4722.0 \\
\hline 25 & 2.6 & 59.86 & 59.96 & 17.73 & 19728 & 1311.6 & -0.0356 & -0.1022 & -1.3 & -5076 & -1775 & -4823.3 \\
\hline 25 & 2.7 & 59.86 & 59.93 & 13.07 & 19255 & 983.3 & -0.0349 & -0.2828 & -46.667 & -4731 & -3283 & -4920.7 \\
\hline 25 & 2.8 & 59.85 & 59.90 & 14.13 & 18814 & 1140.4 & -0.0341 & -0.3 & 10.6 & -4412 & 1571 & 5014.4 \\
\hline 25 & 2.9 & 59.85 & 59.88 & 15.00 & 18401 & 1069.8 & -0.0333 & -0.2233 & 8.667 & -4122 & -706 & 5104.1 \\
\hline 25 & 3 & 59.85 & 59.88 & 14.87 & 18020 & 1076.2 & -0.0322 & 0.0873 & -1.333 & -3817 & 64 & -5189.4 \\
\hline 25 & 3.1 & 59.84 & 59.92 & 14.27 & 17662 & 1016.7 & -0.0315 & 0.3170 & -6.000 & -3577 & -595 & -5269.8 \\
\hline 25 & 3.2 & 59.84 & 59.96 & 13.63 & 17328 & 788.3 & -0.0305 & 0.4055 & -6.333 & -3338 & -2284 & -5345.6 \\
\hline 25 & 3.3 & 60.03 & 60.00 & 13.07 & -99027 & 614.3 & 1.8986 & 0.4477 & -5.667 & -1163600 & -1740 & -5416.3 \\
\hline 25 & 3.4 & 60.04 & 60.03 & 42.93 & -78370 & 938.7 & 0.0745 & 0.3355 & 298.670 & 206580 & 3244 & -5482.9 \\
\hline 25 & 3.5 & 60.04 & 60.04 & 43.93 & -63958 & 957.2 & & & & 120 & 185 & -5543.0 \\
\hline 25 & 3.6 & 60.05 & 60.02 & 13.13 & -62652 & 604.0 & 0.0091 & -0.1928 & -308.000 & 3052 & -3532 & -5593.0 \\
\hline 25 & 3.7 & 59.83 & 59.98 & 14.27 & 15973 & 678.7 & -2.1999 & -0.4145 & 11.333 & 786250 & 748 & -5646.9 \\
\hline 25 & 3.8 & 59.82 & 59.93 & 5.33 & 15755 & 923.3 & -0.0243 & -0.4867 & 10.667 & -2183 & 2445 & -5692.7 \\
\hline 25 & 3.9 & 59.82 & 59.89 & 16.40 & 15550 & 886.7 & -0.0234 & -0.4653 & 10.667 & -2050 & -366 & -5727.6 \\
\hline 25 & 4 & 59.82 & 59.85 & \begin{tabular}{|l|}
17.47 \\
\end{tabular} & 15359 & 965.7 & -0.0224 & -0.3793 & 10.667 & -1907 & 790 & -5767.7 \\
\hline
\end{tabular}


Table A.3. Block Size Metrics for 2PV on 2009HSP Case

\begin{tabular}{|c|c|c|c|c|c|c|c|c|c|c|c|c|}
\hline Gain & $\begin{array}{c}\text { Block } \\
\text { Size } \\
(\mathrm{MW})\end{array}$ & M1 & $\mathrm{M} 2$ & M3 & M4 & M5 & S1 & $\mathrm{S} 2$ & S3 & S4 & S5 & $\begin{array}{l}\text { Total MW } \\
\text { Controlled }\end{array}$ \\
\hline 25 & 0 & 59.92 & 59.94 & \begin{tabular}{|l|}
18.07 \\
\end{tabular} & 36390 & \begin{tabular}{|l|}
937.7 \\
\end{tabular} & 0.0000 & 0.0000 & 0.000 & 0 & 0.0 & -2185.4 \\
\hline 25 & 0.25 & 59.90 & 59.92 & 21.70 & 28385 & 932.7 & -0.0868 & -0.0663 & 14.533 & -32017 & -20.1 & -2001.5 \\
\hline 25 & 0.5 & 59.89 & 59.91 & 24.03 & 24637 & 955.3 & -0.0600 & -0.0387 & 9.333 & -14995 & 90.4 & -1841.5 \\
\hline 25 & 0.75 & 59.88 & 59.91 & 25.83 & 22561 & 981.6 & -0.0418 & -0.0248 & 7.200 & -8303 & 105.3 & -1701.8 \\
\hline 25 & 1 & 59.87 & 59.90 & 26.37 & 21224 & 986.0 & -0.0313 & -0.0142 & 2.133 & -5349 & 17.7 & -1578.0 \\
\hline 25 & 1.25 & 59.86 & 59.90 & 27.33 & 20316 & 1006.0 & -0.0236 & -0.0151 & 3.867 & -3632 & 79.8 & -1515.0 \\
\hline 25 & 1.5 & 59.86 & 59.90 & 28.43 & 19620 & 1018.3 & -0.0196 & -0.0084 & 4.400 & -2783 & 49.3 & -1434.0 \\
\hline 25 & 1.75 & 59.85 & 59.89 & 28.57 & 18965 & 1025.2 & -0.0197 & -0.0097 & 0.533 & -2617 & 27.7 & -1354.5 \\
\hline 25 & 2 & 59.85 & 59.89 & 28.97 & 18461 & 1026.9 & -0.0161 & -0.0047 & 1.600 & -2018 & 6.5 & -1294.0 \\
\hline 25 & 2.25 & 59.85 & 59.89 & 28.20 & 18091 & 1030.7 & -0.0124 & -0.0062 & -3.067 & -1478 & 15.4 & -1246.5 \\
\hline 25 & 2.5 & 59.84 & 59.89 & 27.80 & 17717 & 1038.6 & -0.0131 & -0.0081 & -1.600 & -1497 & 31.6 & -1185.0 \\
\hline 25 & 2.75 & 59.84 & 59.90 & 26.00 & 17455 & 1170.5 & -0.0095 & 0.0560 & -7.200 & -1049 & 527.6 & -1149.5 \\
\hline 25 & 3 & 59.84 & 59.89 & 28.80 & 17272 & \begin{tabular}{|l|}
1059.9 \\
\end{tabular} & -0.0068 & -0.0650 & 11.200 & \begin{tabular}{|l|}
-731 \\
\end{tabular} & -442.4 & -1125.0 \\
\hline 25 & 3.25 & 59.84 & 59.90 & 24.97 & 17083 & 1157.9 & -0.0072 & 0.0422 & -15.333 & -755 & 391.9 & 167.3 \\
\hline 25 & 3.5 & 59.83 & 59.89 & 28.57 & 16896 & 1058.0 & -0.0073 & -0.0495 & 14.400 & -748 & -399.6 & 064.0 \\
\hline 25 & 3.75 & 59.83 & 59.88 & 29.03 & 16645 & 1062.6 & -0.0100 & -0.0052 & 1.867 & -1004 & 18.4 & -1038.8 \\
\hline 25 & 4 & 59.83 & 59.88 & 30.53 & 16434 & 1068.4 & -0.0086 & 0.0 & 000 & $\mid-844$ & 23.3 & -996.0 \\
\hline 25 & 4.25 & 59.83 & 59.88 & 29.53 & 16272 & 1066.6 & -0.0068 & -0.0027 & -4.000 & -650 & -7.1 & -986.0 \\
\hline 25 & 4.5 & 59.83 & 59.88 & 29.37 & 16124 & 1075.1 & -0.0063 & -0.0030 & -0.667 & -589 & 33.7 & -945.0 \\
\hline 25 & 4.75 & 59.82 & 59.88 & 29.87 & 15953 & 1078.8 & -0.0075 & -0.0017 & 2.000 & -687 & 15.0 & -926.3 \\
\hline 25 & 5 & 59.82 & 59.88 & 30.00 & 15820 & 1080.0 & -0.0059 & -0.0008 & 0.533 & -530 & 4.7 & -895.0 \\
\hline 25 & 5.25 & 59.82 & 59.88 & 29.07 & 15785 & 1090.5 & -0.0016 & -0.0002 & -3.733 & -139 & 42.2 & -871.5 \\
\hline 25 & 5.5 & 59.82 & 59.88 & 28.43 & 15656 & 1098.3 & -0.0058 & -0.0047 & -2.533 & -515 & 31.0 & -880.0 \\
\hline 25 & 5.75 & 59.82 & 59.88 & 29.00 & 15513 & 1106.7 & -0.0066 & -0.0040 & 2.267 & -575 & 33.8 & -845.3 \\
\hline 25 & 6 & 59.82 & 59.88 & 31.00 & 15391 & 1119.6 & -0.0057 & 0.0010 & 8.000 & -485 & 51.5 & -822.0 \\
\hline 25 & 6.25 & 59.82 & 59.88 & 30.70 & 15339 & 1120.4 & -0.0025 & -0.0013 & -1.200 & -211 & 3.3 & -806.3 \\
\hline 25 & 6.5 & 59.82 & 59.88 & 29.93 & 15301 & 1121.7 & -0.0018 & -0.00 & -3.067 & -152 & 5.2 & -793.0 \\
\hline 25 & 6.75 & 59.82 & 59.88 & 30.73 & 15224 & 1125.8 & -0.0037 & 0.0004 & 3.200 & -306 & $\begin{array}{ll}16.3 \\
\end{array}$ & -810.0 \\
\hline 25 & 7 & 59.82 & 59.88 & 31.13 & 15127 & 1131.4 & -0.0047 & 0.0018 & 1.600 & -389 & 22.2 & $\begin{array}{l}-791.0 \\
\end{array}$ \\
\hline 25 & 7.25 & 59.81 & 59.88 & 30.37 & 15030 & 1126.9 & -0.0048 & -0.0010 & -3.067 & -389 & -17.7 & -768.5 \\
\hline 25 & 7.5 & 59.81 & 59.88 & 29.40 & 14971 & 1128.8 & -0.0030 & -0.00 & -3.8 & -238 & 7.5 & -742.5 \\
\hline 25 & 7.75 & 59.81 & 59.88 & 28.87 & 14948 & 1129.4 & -0.0011 & -0.0031 & -2.133 & -90 & 2.3 & -759.5 \\
\hline 25 & 8 & 59.81 & 59.88 & 29.47 & 14895 & 1134.8 & -0.0027 & -0.0016 & 2.400 & -212 & 21.7 & -736.0 \\
\hline 25 & 8.25 & 59.81 & 59.88 & 30.70 & 14819 & 1144.0 & -0.0038 & 0.0010 & 4.933 & \begin{tabular}{|l|}
-303 \\
\end{tabular} & 36.8 & -734.3 \\
\hline 25 & 8.5 & 59.81 & 59.88 & \begin{tabular}{|l|}
31.30 \\
\end{tabular} & 14734 & 1146.4 & -0.0044 & 0.0018 & 2.400 & \begin{tabular}{|c|}
-343 \\
\end{tabular} & 9.3 & \begin{tabular}{|l|}
-739.5 \\
\end{tabular} \\
\hline 25 & 8.75 & 59.81 & 59.88 & 30.47 & 14620 & 1143.1 & -0.0059 & -0.0014 & -3.333 & -456 & -13.0 & -726.3 \\
\hline 25 & 9 & 59.81 & 59.88 & 29.77 & 14514 & 1145.7 & -0.0056 & -0.0019 & -2.800 & -421 & 10.1 & -684.0 \\
\hline 25 & 9.25 & 59.81 & 59.88 & 29.77 & 14423 & 1144.6 & -0.0049 & 0.0001 & 0.000 & -365 & -4.2 & -675.3 \\
\hline 25 & $\begin{array}{ll}9.5 \\
\end{array}$ & 59.81 & 59.88 & 28.97 & 14354 & 1148.4 & -0.0038 & & 200 & -278 & 15.1 & -655.5 \\
\hline 25 & 9.75 & 59.80 & 59.88 & 28.40 & 14315 & 1147.1 & -0.0021 & -0.0035 & $\mid-2.267$ & -155 & -5.0 & -643.5 \\
\hline 25 & 10 & 59.80 & 59.88 & 29.03 & 14272 & 1153.5 & -0.0024 & -0.0003 & 2.533 & \begin{tabular}{|c|}
-173 \\
\end{tabular} & 25.6 & -610.0 \\
\hline
\end{tabular}


Table A.4. Gain Metrics with Inhibit Enabled for 2PV on 2009HSP Case

\begin{tabular}{|r|r|r|r|r|r|r|r|r|r|r|r|}
\hline Gain & M1 & M2 & M3 & M4 & M5 & \multicolumn{1}{c|}{ S1 } & \multicolumn{1}{c|}{ S2 } & \multicolumn{1}{c|}{ S3 } & \multicolumn{1}{c|}{ S4 } & \multicolumn{1}{c|}{ S5 } & $\begin{array}{c}\text { Total MW } \\
\text { Controlled }\end{array}$ \\
\hline 5 & 59.84 & 59.91 & 25.13 & 17037 & 1099.3 & 0.0000 & 0.0000 & 0.0000 & 0.0 & 0.0 & -722.2 \\
\hline 10 & 59.88 & 59.93 & 22.50 & 22410 & 1006.2 & 0.0079 & 0.0050 & -0.5267 & 1074.6 & -18.6 & -901.0 \\
\hline 15 & 59.90 & 59.95 & 19.87 & 28129 & 946.6 & 0.0051 & 0.0031 & -0.5267 & 1143.8 & -11.9 & -916.1 \\
\hline 25 & 59.94 & 59.97 & 15.07 & 43388 & 844.2 & 0.0035 & 0.0021 & -0.4800 & 1525.9 & -10.2 & -911.4 \\
\hline 30 & 59.95 & 59.98 & 13.07 & 53092 & 794.5 & 0.0024 & 0.0017 & -0.4000 & 1940.7 & -9.9 & -1034.9 \\
\hline 60 & 60.02 & 60.01 & 15.10 & -171080 & 471.6 & 0.0023 & 0.0011 & 0.0678 & -7472.3 & -10.8 & -1535.9 \\
\hline 90 & 60.03 & 60.02 & 15.87 & -92777 & 266.5 & 0.0005 & 0.0003 & 0.0256 & 2610.0 & -6.8 & -1891.3 \\
\hline 120 & 60.04 & 60.02 & 16.30 & -74127 & 122.6 & 0.0003 & 0.0002 & 0.0144 & 621.7 & -4.8 & -2170.7 \\
\hline
\end{tabular}


Appendix B

Sample Source Code for In-Run EPCL 



\section{Appendix B - Sample Source Code for In-Run EPCL}

A sample listing of an In-run EPCL is shown below. This particular EPCL is set up for a gain of 10 percent with no delay. The sample file is set up so all loads in Zone 999 will be controlled with the exception of the loads at the buses listed in the "Do Not Control" block.

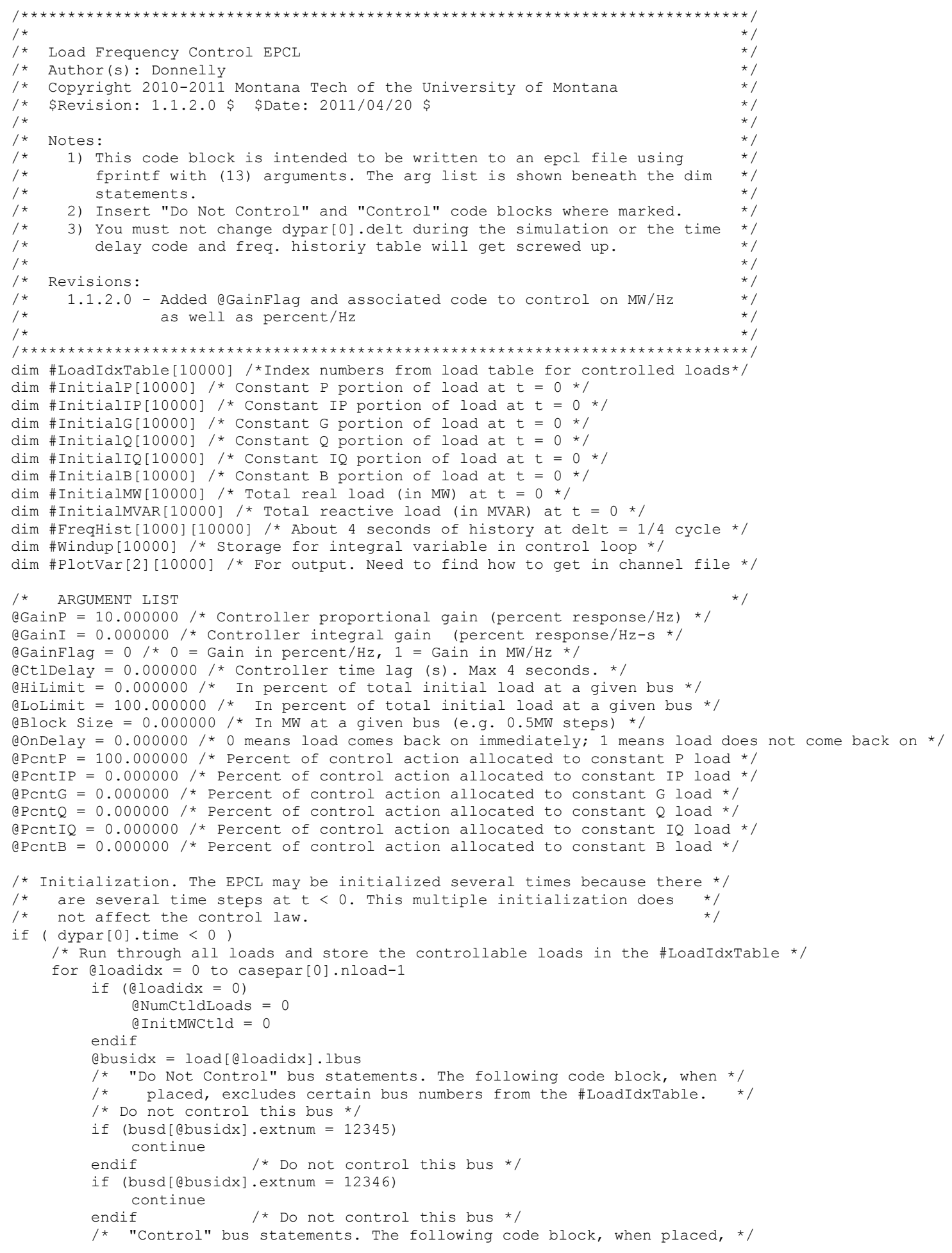




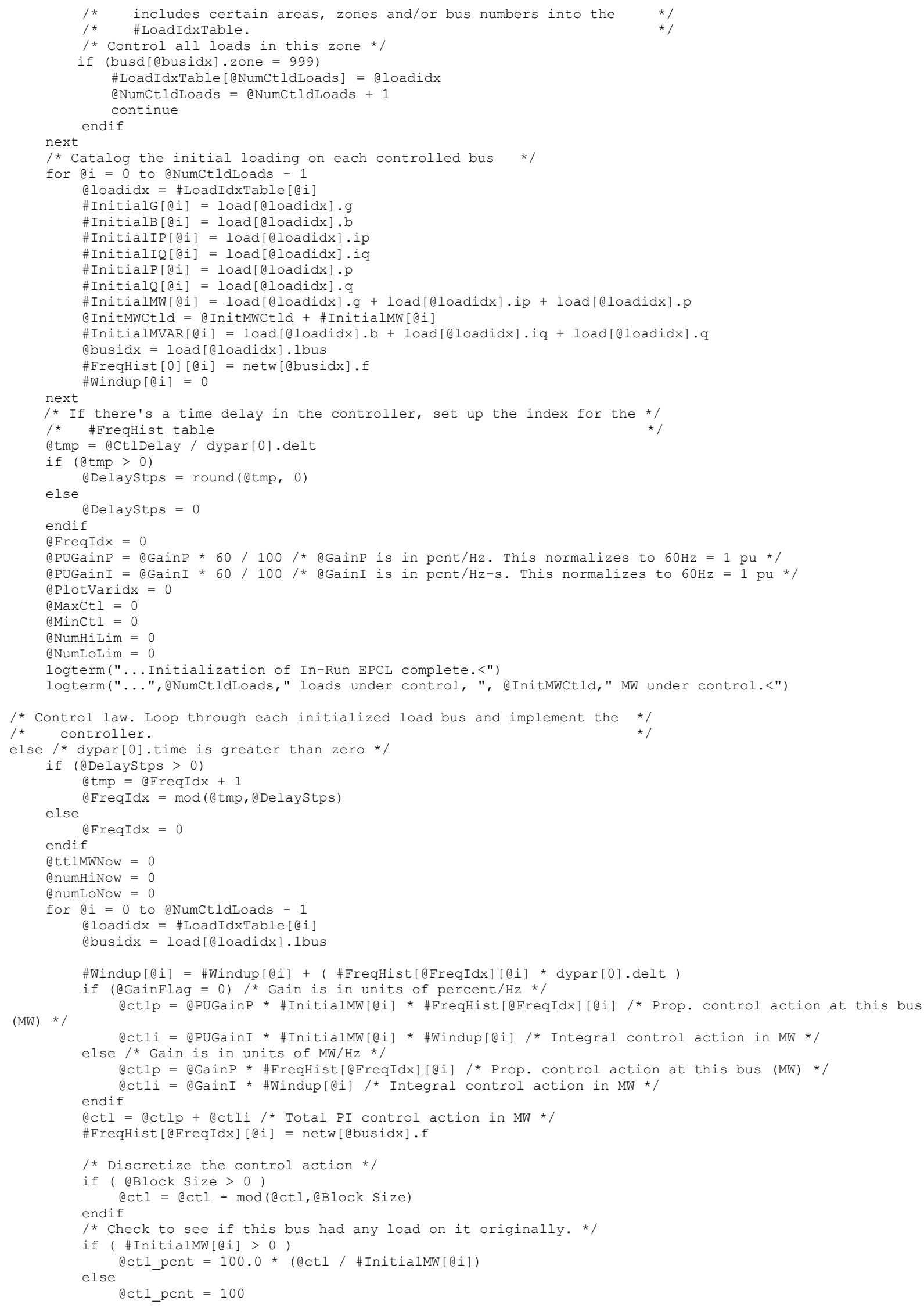


endif

I* Clip the control action */

if ( ectl pent > aHilimit)

@et $1=($ eHiLimit $/ 100) *$ \#InitialMW $[@ i]$

@numHiNow $=$ @numHiNow +1

elseif ( (aLoLimit + ectl pant) $<0$ )

ect $1=0.0-(($ eLoLimit $/ 100) *$ \#InitialmW[@i] $)$

endif @numLoNow $=$ @numLoNow +1

/* Check @onDelay flag to see if this load is eligible for additive control */

@lastStepMW = load[@loadidx].p + load[@loadidx].ip + load[@loadidx].g

if (@onDelay > 0)

if ( (\#InitialMW[ei] + ectl) > @lastStepMW )

endif

endif

/* Allocate between different ZIP components */

/* (MKD: Using IP or G loads could be dangerous.) * /

load[@loadidx].p = \#InitialP[@i] + (@etl * @PcntP / 100)

load[@loadidx].ip = \#InitialIP[@i] + (@etl * @PcntIP / 100)

load[@loadidx].g = \#Initialg[@i] $+($ @etl * @PcntG / 100)

next

attlMWNow $=$ attlMWNow + actl

if (attlMWNow > aMaxctl)

@MaxCtl = @ttlMWNow

elseif (@ttlMWNow $<$ @MinCtl)

endif

@MinCtl = attlMWNow

if (@numHiNow $>$ @NumHiLim)

@NumHiLim $=$ @numHiNow

endif

if (@numLoNow > @NumLoLim)

endif

a NumLoLim $=$ anumLoNow

if (@PlotVaridx $<9999)$

\#PlotVar[0] [@PlotVaridx] = dypar[0].time

\#PlotVar[1] [@PlotVaridx] = @etllMWNow

endif

@PlotVaridx $=$ @PlotVaridx +1

I* Clean up and reporting. Like the initialization, this may execute */

/* multiple times, but it does not affect the control law or output. */

if ( (dypar[0].time + (2*dypar[0].delt)) > dypar[0].tpause )

/* Make a file to store the time domain control action */

\$name = "chans \ctl.dat"

derr $=$ openlog (\$fname)

if (derr $<0)$ endif

logterm("WARNING: Cannot Open Inrun Output File [", \$fname,"]<")

@ tmp = @PlotVaridx -1

for $a i=0$ to atmp

next

logprint (\$fname, \#PlotVar[0] [@i], ">",\#PlotVar[1][@i],"<")

derr $=$ close (\$fname)

/* Make a file to store summary data */

\$name = "inrun.sum"

derr $=$ openlog (\$fname)

if (eerr $<0)$ endif

logterm("WARNING: Cannot Open Inrun Summary File [", \$fname,"]<")

logprint ( $\$$ fname, "Numloads=", enumCtldLoads, "<")

logprint (\$ fname, "TtlMW=", a InitMWCtld," $<"$ )

logprint ( fname, "MaxMW=", @MinCtl, "<")

logprint (\$fname, "Hilim=", @NumHiLim, "<")

logprint ( fname, "Lolim=", eNumLoLim, "<")

endif

derr $=$ close (\$fname)

endif

end 
B. 4 


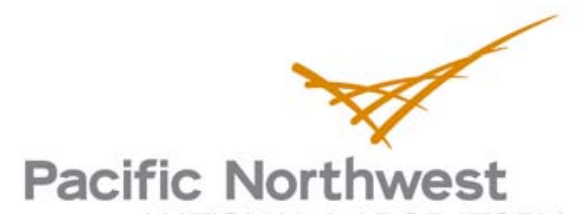

NATIONAL LABORATORY

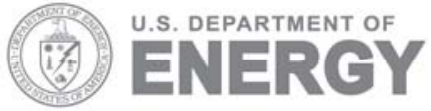

902 Battelle Boulevard

P.O. Box 999

Richland, WA 99352

1-888-375-PNNL (7665)

www.pnl.gov 